Article

\title{
Less Polar Compounds and Targeted Antioxidant Potential (In Vitro and In Vivo) of Codium adhaerens C. Agardh 1822
}

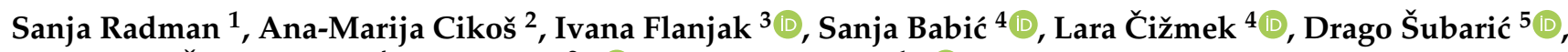 \\ Rozelindra Čož-Rakovac ${ }^{4}$, Stela Jokić ${ }^{2, *}{ }^{\mathbb{D}}$ and Igor Jerković ${ }^{1, * \mathbb{B}}$
}

1 Department of Organic Chemistry, Faculty of Chemistry and Technology, University of Split, Ruđera Boškovića 35, 21000 Split, Croatia; sradman@ktf-split.hr

2 Department of Process Engineering, Faculty of Food Technology, Josip Juraj Strossmayer University of Osijek, Franje Kuhača 18, 31000 Osijek, Croatia; acikos@ptfos.hr

3 Department of Food and Nutrition Research, Faculty of Food Technology, Josip Juraj Strossmayer University of Osijek, Franje Kuhača 18, 31000 Osijek, Croatia; ivana.flanjak@ptfos.hr

4 Laboratory for Aquaculture Biotechnology, Division of Materials Chemistry, Ruđer Bošković Institute, Bijenička cesta 54, 10000 Zagreb, Croatia; sanja.babic@irb.hr (S.B.); lara.cizmek@irb.hr (L.Č.); rozelindra.coz-rakovac@irb.hr (R.Č.-R.)

5 Department of Food Technology, Faculty of Food Technology, Josip Juraj Strossmayer University of Osijek, Franje Kuhača 18, 31000 Osijek, Croatia; dsubaric@ptfos.hr

* Correspondence: sjokic@ptfos.hr (S.J.); igor@ktf-split.hr (I.J.); Tel.: +385-31-224-333 (S.J.); +385-21-329-436 (I.J.)

\section{check for}

updates

Citation: Radman, S.; Cikoš, A.-M.; Flanjak, I.; Babić, S.; Čižmek, L.;

Šubarić, D.; Čož-Rakovac, R.; Jokić, S.; Jerković, I. Less Polar Compounds and Targeted Antioxidant Potential (In Vitro and In Vivo) of Codium adhaerens C. Agardh 1822. Pharmaceuticals 2021, 14, 944. https://doi.org/10.3390/ph14090944

Academic Editors: Marianna Carbone and Maria Letizia Ciavatta

Received: 26 August 2021

Accepted: 18 September 2021

Published: 21 September 2021

Publisher's Note: MDPI stays neutral with regard to jurisdictional claims in published maps and institutional affiliations.

Copyright: (c) 2021 by the authors. Licensee MDPI, Basel, Switzerland. This article is an open access article distributed under the terms and conditions of the Creative Commons Attribution (CC BY) license (https:// creativecommons.org/licenses/by/ $4.0 /)$.
Abstract: Codium adhaerens from the Adriatic Sea (Croatia) was comprehensively investigated regarding less polar compounds for the first time. Although there are several phytochemical studies on $C$. adhaerens from other regions, this is the first report on volatile organic compounds (VOCs) from fresh (FrCa) and air-dried (DrCa) samples. The novelty is also related to its targeted antioxidant potential in vitro and in vivo. The main aims were to: (a) identify and compare VOCs of FrCa and DrCa obtained by headspace solid-phase microextraction (HS-SPME) and hydrodistillation (HD); (b) determine fatty acid (FA) composition of freeze-dried sample (FdCa); (c) determine the composition of less polar fractions of FdCa by high-performance liquid chromatography-high-resolution mass spectrometry with electrospray ionisation (UHPLC-ESI-HRMS); and (d) comprehensively evaluate the antioxidant activity of the fractions by four in vitro assays and in vivo zebrafish model (including embryotoxicity). Significant changes of VOCs were found after air drying. $\omega 6$ FAs were present in higher content than $\omega 3$ FAs indicating $C$. adhaerens as a good source of dietary polyunsaturated FAs. The results obtained in vivo correlate well with in vitro methods and both fractions exerted similar antioxidative responses which is in agreement with the high abundance of present biomolecules with known antioxidant properties (e.g., fucoxanthin, pheophytin $a$, and pheophorbide $a$ ). These results suggest that $C$. adhaerens might be a potent source of natural antioxidants that could be further used in the research of oxidative stress-related diseases.

Keywords: dimethyl sulfide; heptadecane; pheophytin $a$ and its derivatives; pheophorbide $a$ and its derivatives; radical scavenging and antioxidant power; zebrafish model

\section{Introduction}

Interest in using green seaweeds as natural resources has recently increased because of their bioactive constituents, which may be used for medical purposes. Green seaweeds, in general, contain lipids, proteins, peptides, polysaccharides, carotenoids, phenolic compounds, and alkaloids [1,2]. Seaweeds are known to possess nutritional benefits as food and have found much use in industry and medicine for various purposes. Their compounds have complex structures that have shown different biological activities [3-5], including anticancer activity, in several in vitro and in vivo models such as polysaccharides (antibacterial, anticancer, anticoagulant, anti-inflammatory, antioxidant, antiviral, hepatoprotective, 
immunostimulatory, others), phlorotanins (antibacterial, anti-Alzheimer's, anticancer, antidiabetic, anti-inflammatory, antioxidant, antiviral, cytoprotective, hepatoproptective, immunomodulatory, neuroprotective, others), terpenoids (anticancer, antifungal, antioxidant, others), alkaloids (antibacterial, anticancer, anti-inflammatory, antioxidant, antiviral, neuroprotective, others), or carotenoids (anticancer, antidiabetic, anti-inflammatory, antiobesity, antioxidant, neuroprotective, others).

A survey of the literature revealed different phytochemical studies on Codium adhaerens. Bioactive compounds present in its ethanol extract were analysed by gas chromatographymass spectrometry (GC-MS) after the silylation [6]. Proline, mannitol and $\beta$-sitosterol were found. Concerning fatty acids, it was found that C. adhaerens contained palmitic, $\alpha$ linolenic, oleic, stearic, arachidonic, eicosapentaenoic and arachidic acids. Relative pigment composition of $C$. adhaerens was determined by the cellulose thin-layer chromatogram and chlorophyll $a$, chlorophyll $b$ and siphonaxanthin were determined [7]. Amino acid composition was determined before and after the hydrolysis in C. adhaerens ethanol extract and alanine, asparagine, aspartic acid, glutamic acid, glutamine, glycine, isoleucine, proline, serine, taurine, threonine and valine were found [8]. The sterol profiles were evaluated by the procedure involving alkaline hydrolysis and extraction followed by separation by reversed-phase high-performance liquid chromatography (HPLC)-diode array detection (HPLC-DAD) and C. adhaerens contained desmosterol, ergosterol, fucosterol, cholesterol, campesterol, stigmasterol and $\beta$-sitosterol [9]. Recently, a great deal of interest has been directed toward the isolation of novel sulfated polysaccharides (SPs) from marine green algae because of their numerous health-beneficial effects. Green seaweeds are known to synthesise large quantities of SPs, and arabinose was reported to be the major monosaccharide of SPs from C. adhaerens $[2,10]$. These SPs exhibit many beneficial biological activities, such as anticoagulant, antiviral, antioxidative, antitumor, immunomodulating, antihyperlipidemic and antihepatotoxic activities [2,10].

However, no reports on the volatile organic compounds (VOCs) from C. adhaerens were found, and therefore we decided to investigate them in detail. Since our previous studies [11,12] indicated great diversity of VOCs from fresh and air-dried samples (including Codium bursa) we decided to research the variability of VOCs of $C$. adhaerens from fresh (FrCa) and air-dried (DrCa) samples. In addition, VOCs have often been related to less polar constituents, whose comprehensive antioxidant potential from C. adhaerens (in vivo and in vitro) was targeted in the current research for the first time. The main goals of the present research were to: (a) identify and compare VOCs of FrCa and DrCa obtained by headspace solid-phase microextraction (HS-SPME) and hydrodistillation (HD) followed by analysis with gas chromatography and mass spectrometry (GC-MS); (b) determine fatty acid composition of freeze-dried sample ( $\mathrm{FdCa}$ ) after derivatisation as methyl esters by gas chromatography analysis with flame-ionisation detector (GC-FID); (c) determine the composition of less polar fractions of $\mathrm{FdCa}$ by high-performance liquid chromatography-high-resolution mass spectrometry with electrospray ionisation (UHPLC-ESI-HRMS); (d) comprehensively evaluate the antioxidant activity of the fractions by four in vitro assays (Folin-Ciocalteu, reduction of radical cation $\mathrm{ABTS}^{\bullet+}$, 2,2-diphenyl-1-picryl-hydrazyl-hydrate (DPPH) assay, and ferric reducing antioxidant power (FRAP)) and in vivo zebrafish model (including embryotoxicity).

\section{Results and Discussion}

\subsection{Headspace Composition}

Two fibres of different polarity (divinylbenzene/carboxene/polydimethylsiloxane (DVB/CAR/PDMS) and polydimethylsiloxane/divinylbenzene (PDMS/DVB)) were used for HS-SPME. By this approach, more complete headspace profile could be achieved due to combination of the fibres of different polarities. In FrCa headspace (HS-FrCa) $91.52 \%$ of VOCs were identified in total by HS-SPME with DVB/CAR/PDMS fibre and $83.25 \%$ by HS-SPME with PDMS/DVB fibre, Table 1. 
Table 1. The volatile compounds from Codium adhaerens isolated by headspace solid-phase microextraction (HS-SPME) and analysed by gas chromatography-mass spectrometry (GC-MS): (I-fresh C. adhaerens extracted by DVB/CAR/PDMS fibre, II-air-dried C. adhaerens extracted by DVB/CAR/PDMS fibre, III-fresh C. adhaerens extracted by PDMS/DVB fibre, IV-air-dried C. adhaerens extracted by PDMS/DVB fibre).

\begin{tabular}{|c|c|c|c|c|c|c|}
\hline \multirow{2}{*}{ No. } & \multirow{2}{*}{ Compound } & \multirow{2}{*}{ RI } & \multicolumn{4}{|c|}{ Area $(\%) \pm \mathrm{SD}$} \\
\hline & & & I & II & III & IV \\
\hline 1 & Dimethyl sulfide & $<900$ & $54.25 \pm 2.13$ & $0.42 \pm 0.03$ & $37.83 \pm 1.19$ & $0.62 \pm 0.03$ \\
\hline 2 & Iodomethane & $<900$ & - & $2.0 \pm 0.07$ & - & $2.48 \pm 0.09$ \\
\hline 3 & 2-Ethylfuran & $<900$ & $4.52 \pm 0.18$ & - & - & - \\
\hline 4 & Pentanal & $<900$ & - & $0.20 \pm 0.07$ & - & $0.11 \pm 0.04$ \\
\hline 5 & Hexanal & $<900$ & $0.65 \pm 0.03$ & $1.67 \pm 0.09$ & $2.46 \pm 0.93$ & $2.59 \pm 0.36$ \\
\hline 6 & Dimethylsulfoxide & $<900$ & - & $19.50 \pm 0.49$ & - & $12.38 \pm 0.62$ \\
\hline 7 & Heptanal & 902 & - & $0.95 \pm 0.16$ & $0.90 \pm 0.35$ & $2.29 \pm 0.37$ \\
\hline 8 & Diodomethane & 923 & - & $0.26 \pm 0.01$ & - & $0.40 \pm 0.10$ \\
\hline 9 & 1-Iodopentane & 927 & - & $0.31 \pm 0.04$ & - & $0.902 \pm 0.21$ \\
\hline 10 & $\alpha$-Pinene & 941 & - & $0.25 \pm 0.05$ & - & $0.73 \pm 0.21$ \\
\hline 11 & (2E)-Hept-2-enal & 964 & - & - & - & $0.86 \pm 0.30$ \\
\hline 12 & Benzaldehyde & 966 & $12.84 \pm 0.50$ & $3.64 \pm 0.04$ & $12.22 \pm 5.62$ & $2.06 \pm 0.13$ \\
\hline 13 & Oct-1-en-3-ol & 985 & $2.45 \pm 0.10$ & $1.95 \pm 0.01$ & $2.40 \pm 0.68$ & $1.27 \pm 0.11$ \\
\hline 14 & Phenol & 987 & - & - & $0.64 \pm 0.08$ & $1.20 \pm 0.17$ \\
\hline 15 & 6-Methylhept-5-en-2-one & 989 & $0.63 \pm 0.02$ & $0.64 \pm 0.01$ & $0.43 \pm 0.16$ & $0.63 \pm 0.01$ \\
\hline 16 & 2-Pentylfuran & 996 & $1.07 \pm 0.04$ & $0.90 \pm 0.00$ & $0.58 \pm 0.22$ & $1.80 \pm 0.20$ \\
\hline 17 & Octanal & 1004 & - & $0.75 \pm 0.05$ & $0.73 \pm 0.28$ & $1.15 \pm 0.13$ \\
\hline 18 & $(2 E, 4 E)$-Hepta-2,4-dienal & 1015 & - & $0.37 \pm 0.03$ & $1.02 \pm 0.42$ & $0.80 \pm 0.10$ \\
\hline 19 & 2-Ethylhexan-1-ol & 1033 & - & - & - & $0.89 \pm 0.24$ \\
\hline 20 & $p$-Cymene & 1034 & - & $0.35 \pm 0.17$ & - & - \\
\hline 21 & Limonene & 1037 & $0.60 \pm 0.02$ & $0.75 \pm 0.25$ & - & $0.68 \pm 0.26$ \\
\hline 22 & Benzyl alcohol & 1042 & $2.22 \pm 0.09$ & $6.71 \pm 0.12$ & $1.60 \pm 0.44$ & $12.35 \pm 0.88$ \\
\hline 23 & $(2 E)$-Oct-2-enal & 1063 & - & $0.77 \pm 0.01$ & $1.29 \pm 0.66$ & $1.25 \pm 0.17$ \\
\hline 24 & $(3 E, 5 E)$-Octa-3,5-dien-2-one & 1097 & - & $1.20 \pm 0.04$ & & $0.93 \pm 0.03$ \\
\hline 25 & Linalool & 1102 & - & $0.22 \pm 0.02$ & $0.41 \pm 0.15$ & $0.53 \pm 0.21$ \\
\hline 26 & Nonanal & 1107 & - & $0.89 \pm 0.10$ & $1.41 \pm 0.55$ & $2.20 \pm 0.28$ \\
\hline 27 & $(2 E, 4 E)$-Octa-2,4-dienal & 1114 & - & & $1.36 \pm 0.19$ & \\
\hline 28 & 2,6-Dimethylcyclohexanol & 1115 & - & $0.61 \pm 0.02$ & & $0.61 \pm 0.14$ \\
\hline 29 & $\begin{array}{c}\text { 6-[(Z)-1-Butenyl]-1,4-cycloheptadiene } \\
\text { (Dictyopterene D) }\end{array}$ & 1159 & - & - & $0.72 \pm 0.28$ & - \\
\hline 30 & (2Z)-Non-2-enal & 1165 & - & $0.61 \pm 0.11$ & - & $2.47 \pm 0.44$ \\
\hline 31 & Indole & 1297 & - & $0.27 \pm 0.05$ & $0.86 \pm 0.34$ & $0.37 \pm 0.15$ \\
\hline 32 & Tridecane & 1300 & $0.93 \pm 0.04$ & $2.96 \pm 0.39$ & $0.71 \pm 0.13$ & $1.81 \pm 0.06$ \\
\hline 33 & $\alpha$-Ionone & 1433 & $2.63 \pm 0.10$ & $1.18 \pm 0.01$ & $2.52 \pm 0.78$ & $1.19 \pm 0.29$ \\
\hline 34 & Dimethyl phthalate & 1461 & - & $0.44 \pm 0.01$ & - & $0.59 \pm 0.04$ \\
\hline 35 & $(E)$ - $\beta$-Farnesene & 1463 & - & - & $0.89 \pm 0.19$ & $0.31 \pm 0.03$ \\
\hline 36 & $\beta$-Ionone & 1487 & $1.21 \pm 0.05$ & $0.61 \pm 0.04$ & - & - \\
\hline
\end{tabular}


Table 1. Cont.

\begin{tabular}{|c|c|c|c|c|c|c|}
\hline \multirow{2}{*}{ No. } & \multirow{2}{*}{ Compound } & \multirow{2}{*}{ RI } & \multicolumn{4}{|c|}{ Area $(\%) \pm \mathrm{SD}$} \\
\hline & & & $\mathbf{I}$ & II & III & IV \\
\hline 37 & $\alpha$-Curcumene & 1488 & - & - & $0.58 \pm 0.22$ & $0.29 \pm 0.03$ \\
\hline 38 & Pentadec-1-ene & 1495 & - & $0.51 \pm 0.04$ & - & $0.42 \pm 0.08$ \\
\hline 39 & Pentadecane & 1500 & $0.48 \pm 0.02$ & $4.36 \pm 0.01$ & $0.47 \pm 0.18$ & $2.30 \pm 0.48$ \\
\hline 40 & $\begin{array}{l}\text { 5,6,7,7a-Tetrahydro-4,4,7a-trimethyl- } \\
\text { 2(4H)-benzofuranone }\end{array}$ & 1534 & $0.39 \pm 0.02$ & $0.40 \pm 0.07$ & - & - \\
\hline 41 & Dihydroactinolide & 1534 & - & - & - & $0.33 \pm 0.13$ \\
\hline 42 & Heptadecane & 1700 & $6.65 \pm 0.26$ & $26.61 \pm 0.90$ & $11.22 \pm 3.01$ & $23.12 \pm 1.86$ \\
\hline
\end{tabular}

In DrCa headspace (HS-DrCa), 82.08\% (DVB/CAR/PDMS fibre) and 84.91\% (PDMS/DVB fibre) of VOCs were identified in total by HS-SPME. The majority of identified VOCs in HS-FrCa belong to sulphur compounds, organoiodines, esters, and lactones, and in HSDrCa the majority of VOCs were comprised of saturated hydrocarbons. The dominant compound in HS-FrCa was dimethyl disulfide, DMS, (54.25\%—DVB/CAR/PDMS fibre; $37.83 \%-P D M S / D V B$ fibre). Distribution of the compound structural groups in HS-FrCa and HS-DrCa is presented in Figure 1.

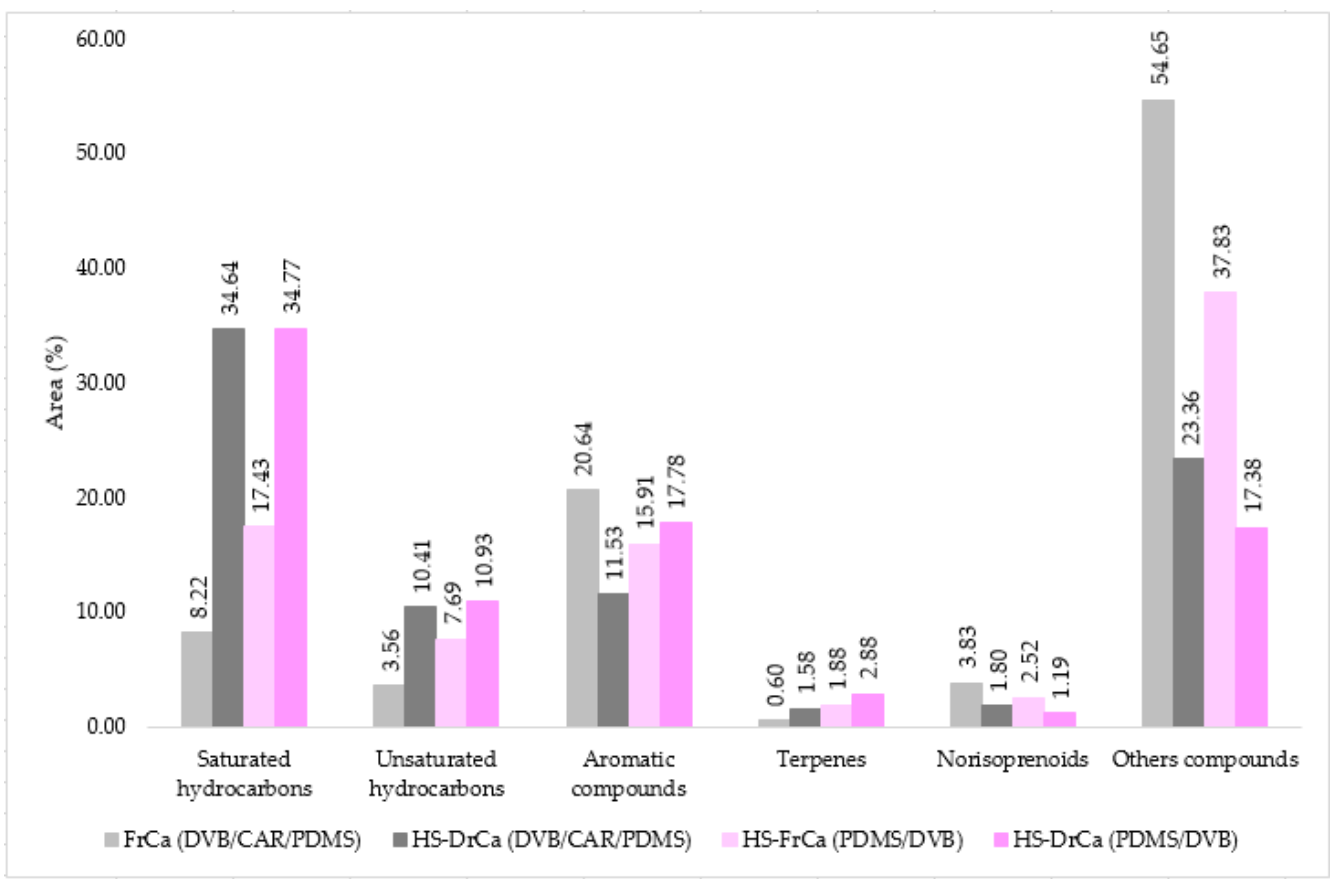

Figure 1. The VOCs from C. adhaerens sorted by structural groups extracted by HS-SPME.

The headspace chemical profile showed that DMS was the main identified compound in the seagrass Posedonia oceanica (59.3\%), green alga Flavellia petiolata $(22.2 \%)$, brown alga Halopteris filicina (12.8\%) [13] and green alga C. bursa (56.51\%) [12]. It is known that DMS can be easily oxidised into dimethyl sulfoxide (DMSO) in the contact with air [14,15] which can be noticed as the result of air-drying of $C$. adhaerens. Namely, the amount of DMS decreased 129.2 times (DVB/CAR/PDMS fibre) or 61.0 times (PDMS/DVB fibre) in the sample after air-drying, resulting in the detection of highly abundant DMSO $(19.50 \% ; 12.38 \%)$. The second dominant compound in HS-FrCa was the aromatic compound benzaldehyde $(12.84 \% ; 12.22 \%)$, which decreased 3.5 or 5.9 times in HS-DrCa $(3.64 \% ; 2.06 \%)$. The loss of benzaldehyde during air-drying could be a consequence of its higher volatility in com- 
parison to benzyl alcohol rather than its oxidation to benzoic acid that cannot be found by HS-SPME, and was also not identified by HD. The percentage of aromatic compound benzyl alcohol, as the second most abundant compound is $\mathrm{HS}-\mathrm{DrCa}$, increased 3.0 (or 7.7) times in HS-DrCa from $2.22 \%$ (or $1.60 \%$ ) to $6.71 \%$ (or $12.35 \%$ ). Its increase during drying could be connected to lignin degradation, since lignin-related compounds were found in the plant cell walls of Codium fragile [16]. In fact, the green algae extracellular coverings, including cell walls, showed remarkable structural and biochemical similarity to the land plant cell walls containing assemblages of polymers with notable similarity to pectins, cellulose, hemicelluloses, extensin, arabinogalactan proteins (AGPs), and lignin [17]. Aliphatic hydrocarbon heptadecane was the major compound in HS-DrCa. Heptadecane is known to be the most abundant alkane in green algae $[18,19]$. The abundance of heptadecane in HS-DrCa was higher with DVB/CAR/PDMS fibre (26.61\%) with respect to PDMS/DVB fibre $(23.12 \%)$. The increment of heptadecane with air-drying was noticed (4.0 timesDVB/CAR/PDMS fibre; 2.1 times-PDMS/DVB fibre), probably as a result of fatty acid decarboxylation [20]. Additionally, two saturated aliphatic hydrocarbons were noticed to increase by a great percentage after air-drying (probably due to fatty acids degradation): tridecane (3.2 times; or 2.5 times) and pentadecane (9.1 times; or 4.9 times). In HS-DrCa, three organoiodines were identified (more abundant with PDMS/DVB fibre): iodomethane $(2.00 \%$; or $2.48 \%)$, diiodomethane $(0.26 \%$; or $0.40 \%)$ and 1 -iodopentane $(0.31 \%$; or $0.90 \%)$; while in HS-FrCa none of them were detected. Marine macroalgae showed great potency of linking halogen ions resulting in halogenated secondary metabolites formation [21]. Palmer et al. [22] showed that different oxidative stress, including desiccation, increased the volatile iodinated compounds in brown alga Laminaria digitata. Bravo-Linares et al. [23] investigated the production of VOCs in response to environmental stresses and they have found that all tested algae showed greater release of iodinated compounds under stress, especially iodomethane. Even though the brown algae showed the greatest release of iodinated compounds (iodoethane, 2-iodopropane, 1-iodobutane, diiodomethane and iodomethane), the green algae Ulva lactuca and Enteromorpha sp. also contained iodinated compounds that increased during drying process. In the chemical profile of brown alga Aschophyllum nodosum headspace 1-iodopentane ( $0.8 \%$ ) was identified as the only organoiodine [13]. It has already been indicated that the volatile halogenated compounds may be involved in the defence mechanism of many different organisms including algae [24]. The enzymes involved in halogenated organic compounds synthesis are haloperoxidases [24].

\subsection{Volatile Oil Composition}

In the hydrodistillate of FrCa (HD-FrCa) $87.16 \%$ and of $\mathrm{DrCa}$ (HD-DrCa) $97.96 \%$ of total ion chromatogram area were identified in total, Table 2.

Table 2. The volatile compounds from C. adhaerens isolated by hydrodistillation (HD) and analysed by gas chromatographymass spectrometry (GC-MS): (VI—hydrodistillate of fresh C. adhaerens, VII-hydrodistillate of air-dried C. adhaerens).

\begin{tabular}{|c|c|c|c|c|}
\hline \multirow{2}{*}{ No. } & \multirow{2}{*}{ Compound } & \multirow{2}{*}{ RI } & \multicolumn{2}{|c|}{ Area $(\%) \pm \mathrm{SD}$} \\
\hline & & & VI & VII \\
\hline 1 & Furan-2-carbaldehyde & $<900$ & $0.06 \pm 0.01$ & $0.11 \pm 0.04$ \\
\hline 2 & $(2 E)$-Hex-2-enal & $<900$ & $0.36 \pm 0.11$ & $0.13 \pm 0.05$ \\
\hline 3 & 4-Methyloctane & $<900$ & $0.15 \pm 0.04$ & $0.36 \pm 0.19$ \\
\hline 4 & Hexan-1-ol & $<900$ & $0.21 \pm 0.06$ & - \\
\hline 5 & Heptan-3-one & $<900$ & $0.07 \pm 0.02$ & $0.08 \pm 0.00$ \\
\hline 6 & 5-Methylhexan-2-one & $<900$ & - & $0.31 \pm 0.08$ \\
\hline 7 & Non-1-ene & $<900$ & $0.05 \pm 0.01$ & - \\
\hline
\end{tabular}


Table 2. Cont.

\begin{tabular}{|c|c|c|c|c|}
\hline \multirow{2}{*}{ No. } & \multirow{2}{*}{ Compound } & \multirow{2}{*}{ RI } & \multicolumn{2}{|c|}{ Area $(\%) \pm S D$} \\
\hline & & & VI & VII \\
\hline 8 & $(2 E, 4 E)$-Hexa-2,4-diene & $<900$ & - & $0.04 \pm 0.01$ \\
\hline 9 & Heptanal & 902 & $0.18 \pm 0.04$ & $0.51 \pm 0.11$ \\
\hline 10 & Hepta-2,4-dien-1-al * & 907 & - & $0.07 \pm 0.02$ \\
\hline 11 & Diodomethane & 923 & - & $0.16 \pm 0.00$ \\
\hline 12 & 1-Iodopentane & 927 & - & $0.08 \pm 0.03$ \\
\hline 13 & $\alpha$-Pinene & 941 & - & $0.03 \pm 0.01$ \\
\hline 14 & Benzaldehyde & 966 & $0.40 \pm 0.10$ & $0.77 \pm 0.14$ \\
\hline 15 & Sabinene & 979 & - & $0.03 \pm 0.01$ \\
\hline 16 & Oct-1-en-3-ol & 985 & $0.10 \pm 0.02$ & $0.24 \pm 0.02$ \\
\hline 17 & Octan-2,3-dione & 986 & - & $0.44 \pm 0.16$ \\
\hline 18 & Phenol & 987 & $0.13 \pm 0.04$ & $0.08 \pm 0.03$ \\
\hline 19 & 6-Methylhept-5-en-2-one & 989 & - & $0.13 \pm 0.04$ \\
\hline 20 & 2-Pentylfuran & 996 & $0.21 \pm 0.06$ & $0.90 \pm 0.04$ \\
\hline 21 & $\begin{array}{l}\text { 2,4,6-Trimethylpyridine } \\
(\alpha \text {-Collidine })\end{array}$ & 997 & $0.09 \pm 0.03$ & $0.19 \pm 0.09$ \\
\hline 22 & Octanal & 1004 & $0.10 \pm 0.05$ & $0.28 \pm 0.06$ \\
\hline 23 & $(2 E, 4 E)$-Hepta-2,4-dienal & 1015 & $0.08 \pm 0.00$ & $0.13 \pm 0.05$ \\
\hline 24 & 2-Ethylhexan-1-ol & 1033 & $0.11 \pm 0.03$ & $0.25 \pm 0.09$ \\
\hline 25 & Benzyl alcohol & 1042 & $0.65 \pm 0.14$ & $0.67 \pm 0.04$ \\
\hline 26 & 2,4,4-Trimethylcyclohex-2-en-1-ol & 1059 & $0.15 \pm 0.03$ & $0.35 \pm 0.01$ \\
\hline 27 & $(2 E)$-Oct-2-enal & 1063 & $0.27 \pm 0.06$ & $0.33 \pm 0.02$ \\
\hline 28 & Acetophenone & 1072 & $0.11 \pm 0.03$ & $0.17 \pm 0.01$ \\
\hline 29 & Octylcyclopropane & 1074 & $0.19 \pm 0.08$ & $0.17 \pm 0.06$ \\
\hline 30 & Nonan-2-one & 1095 & - & $0.07 \pm 0.03$ \\
\hline 31 & $(3 E, 5 E)$-Octa-3,5-dien-2-one & 1097 & - & $0.26 \pm 0.01$ \\
\hline 32 & Linalool & 1102 & $0.13 \pm 0.03$ & $0.07 \pm 0.02$ \\
\hline 33 & Nonanal & 1107 & $0.17 \pm 0.02$ & $0.25 \pm 0.02$ \\
\hline 34 & 2,6-Dimethylcyclohexanol & 1115 & $0.13 \pm 0.03$ & $0.28 \pm 0.05$ \\
\hline 35 & $\alpha$-Cyclocitral & 1122 & - & $0.03 \pm 0.01$ \\
\hline 36 & $\begin{array}{l}\text { 2-Hydroxy-3,5,5-trimethylcyclohex-2-en-1-one } \\
\text { (2-Hydroxyisophorone) }\end{array}$ & 1126 & - & $0.03 \pm 0.01$ \\
\hline 37 & 4-Ketoisophorone & 1150 & $0.18 \pm 0.03$ & $0.13 \pm 0.02$ \\
\hline 38 & (2Z)-Non-2-enal & 1165 & $0.16 \pm 0.03$ & $0.22 \pm 0.04$ \\
\hline 39 & Benzylmethylsulfide & 1171 & - & $0.20 \pm 0.01$ \\
\hline 40 & 2,4-Dimethylbenzaldehyde & 1180 & - & $0.09 \pm 0.03$ \\
\hline 41 & Decan-2-one & 1196 & - & $0.48 \pm 0.11$ \\
\hline 42 & Safranal & 1204 & - & $0.36 \pm 0.06$ \\
\hline 43 & Decanal & 1209 & $0.14 \pm 0.02$ & $0.08 \pm 0.04$ \\
\hline 44 & $\beta$-Cyclocitral & 1226 & - & $0.15 \pm 0.05$ \\
\hline 45 & Benzothiazole & 1229 & - & $0.07 \pm 0.02$ \\
\hline
\end{tabular}


Table 2. Cont.

\begin{tabular}{|c|c|c|c|c|}
\hline \multirow{2}{*}{ No. } & \multirow{2}{*}{ Compound } & \multirow{2}{*}{ RI } & \multicolumn{2}{|c|}{ Area $(\%) \pm \mathrm{SD}$} \\
\hline & & & VI & VII \\
\hline 46 & $\beta$-Cyclohomocitral & 1263 & $0.11 \pm 0.04$ & $0.11 \pm 0.00$ \\
\hline 47 & $\delta$-Octalactone & 1264 & $0.18 \pm 0.03$ & - \\
\hline 48 & (3Z)-Tridec-3-ene & 1295 & $0.12 \pm 0.03$ & - \\
\hline 49 & 1H-Indole & 1297 & $0.30 \pm 0.07$ & $1.37 \pm 0.19$ \\
\hline 50 & Tridecane & 1300 & $2.38 \pm 0.52$ & $1.04 \pm 0.21$ \\
\hline 51 & Undecanal & 1311 & $0.14 \pm 0.05$ & - \\
\hline 52 & $(2 E, 4 E)$-Deca-2,4-dienal & 1321 & $0.15 \pm 0.00$ & $0.12 \pm 0.03$ \\
\hline 53 & $\begin{array}{c}\text { 1,1,6-Trimethyl-2H-naphthalene } \\
\text { (3,4-Dehydroionene) }\end{array}$ & 1358 & $0.09 \pm 0.02$ & $0.05 \pm 0.02$ \\
\hline 54 & Hexahydropseudoionone & 1409 & $0.16 \pm 0.03$ & $0.08 \pm 0.03$ \\
\hline 55 & $\alpha$-Ionone & 1433 & $0.61 \pm 0.12$ & $5.84 \pm 0.72$ \\
\hline 56 & 2-Methoxynaphthalene & 1453 & $0.09 \pm 0.03$ & $0.05 \pm 0.02$ \\
\hline 57 & (Z)-Geranylacetone & 1459 & - & $0.48 \pm 0.06$ \\
\hline 58 & (5E)-Dodec-5-en-1-ol & 1467 & $0.23 \pm 0.04$ & - \\
\hline 59 & $\beta$-Ionone & 1487 & - & $1.22 \pm 0.45$ \\
\hline 60 & $\beta$-Ionene & 1490 & $0.85 \pm 0.14$ & $1.81 \pm 0.33$ \\
\hline 61 & Pentadec-1-ene & 1495 & $2.12 \pm 0.36$ & $0.67 \pm 0.12$ \\
\hline 62 & Pentadecane & 1500 & $3.25 \pm 0.67$ & $1.84 \pm 0.27$ \\
\hline 63 & (7E)-Pentadec-7-ene & 1509 & $0.26 \pm 0.04$ & - \\
\hline 64 & Tridecanal & 1515 & $0.21 \pm 0.01$ & $0.09 \pm 0.03$ \\
\hline 65 & Dihydroactinolide & 1534 & - & $0.45 \pm 0.08$ \\
\hline 66 & Tetradecan-2-one & 1566 & $0.21 \pm 0.06$ & $0.16 \pm 0.01$ \\
\hline 67 & Dodecanoic acid & 1573 & $0.52 \pm 0.06$ & $0.19 \pm 0.07$ \\
\hline 68 & Tridecan-1-ol & 1580 & $0.52 \pm 0.02$ & $0.37 \pm 0.06$ \\
\hline 69 & Diethyl phthalate & 1599 & - & $0.32 \pm 0.12$ \\
\hline 70 & Hexadecane & 1600 & $0.40 \pm 0.15$ & $0.14 \pm 0.05$ \\
\hline 71 & Tetradecanal & 1617 & $0.54 \pm 0.00$ & $0.14 \pm 0.03$ \\
\hline 72 & Benzophenone & 1630 & - & $0.23 \pm 0.08$ \\
\hline 73 & $\begin{array}{c}\text { (6Z)-Dodec-6-en-4-olide } \\
((Z)-6-\gamma \text {-Dodecenolactone) }\end{array}$ & 1661 & $2.46 \pm 0.17$ & $0.85 \pm 0.09$ \\
\hline 74 & Tetradecan-1-ol & 1683 & $3.55 \pm 0.38$ & $2.16 \pm 0.26$ \\
\hline 75 & (8E)-Heptadec-8-ene & 1690 & $0.44 \pm 0.06$ & $0.30 \pm 0.11$ \\
\hline 76 & Heptadec-1-ene & 1697 & $2.80 \pm 0.42$ & $1.61 \pm 0.13$ \\
\hline 77 & Heptadecane & 1700 & $29.32 \pm 6.86$ & $12.29 \pm 0.25$ \\
\hline 78 & (3Z)-Heptadec-3-ene & 1709 & $0.32 \pm 0.05$ & $0.11 \pm 0.04$ \\
\hline 79 & Pentadecanal & 1720 & $0.56 \pm 0.03$ & $0.17 \pm 0.06$ \\
\hline 80 & 7-Methylheptadecane & 1750 & $0.31 \pm 0.00$ & $0.70 \pm 0.02$ \\
\hline 81 & Tetradecanoic acid & 1772 & $2.43 \pm 0.39$ & $0.24 \pm 0.04$ \\
\hline 82 & Octadec-1-ene & 1790 & $0.19 \pm 0.07$ & - \\
\hline 83 & Octadecane & 1800 & $0.29 \pm 0.03$ & $0.09 \pm 0.03$ \\
\hline
\end{tabular}


Table 2. Cont.

\begin{tabular}{|c|c|c|c|c|}
\hline \multirow{2}{*}{ No. } & \multirow{2}{*}{ Compound } & \multirow{2}{*}{ RI } & \multicolumn{2}{|c|}{ Area $(\%) \pm S D$} \\
\hline & & & VI & VII \\
\hline 84 & Hexadecanal & 1822 & $1.32 \pm 0.08$ & $0.19 \pm 0.02$ \\
\hline 85 & Neophytadiene & 1845 & $0.16 \pm 0.06$ & $0.10 \pm 0.04$ \\
\hline 86 & Hexahydrofarnesyl acetone (Phytone) & 1852 & $2.03 \pm 0.10$ & $5.81 \pm 0.48$ \\
\hline 87 & p-Cumylphenol & 1857 & $0.27 \pm 0.04$ & - \\
\hline 88 & (9Z)-Hexadeca-1,9-diene & 1866 & $0.49 \pm 0.13$ & $6.53 \pm 0.48$ \\
\hline 89 & (11Z)-Hexadec-11-enal & 1870 & $0.21 \pm 0.03$ & $0.25 \pm 0.04$ \\
\hline 90 & Diisobutyl phthalate & 1874 & $0.42 \pm 0.02$ & $1.10 \pm 0.05$ \\
\hline 91 & $(9 E)$-Nonadec-9-ene & 1879 & $0.49 \pm 0.01$ & $0.22 \pm 0.03$ \\
\hline 92 & Hexadecan-1-ol & 1886 & $1.63 \pm 0.25$ & $1.57 \pm 0.50$ \\
\hline 93 & Nonadec-1-ene & 1898 & $0.25 \pm 0.04$ & $0.10 \pm 0.04$ \\
\hline 94 & Nonadecane & 1900 & $0.70 \pm 0.08$ & $0.33 \pm 0.00$ \\
\hline 95 & Hexadecanoic acid & 1981 & $8.62 \pm 1.61$ & $10.55 \pm 1.40$ \\
\hline 96 & Cyclooctasulfur & 2018 & $0.57 \pm 0.05$ & $0.42 \pm 0.16$ \\
\hline 97 & Octadecanal & 2025 & $0.71 \pm 0.08$ & $0.22 \pm 0.03$ \\
\hline 98 & Methyl octadecyl ether & 2034 & $2.06 \pm 0.24$ & $1.22 \pm 0.08$ \\
\hline 99 & Octadecan-1-ol & 2086 & $0.51 \pm 0.13$ & $1.75 \pm 0.90$ \\
\hline 100 & (E)-Phytol & 2119 & $5.00 \pm 1.14$ & $16.99 \pm 2.69$ \\
\hline 101 & (9Z)-octadec-9-enoic acid (Oleic acid) & 2146 & $0.49 \pm 0.27$ & $2.53 \pm 0.37$ \\
\hline 102 & (5E)-Icos-5-ene & 2293 & $0.65 \pm 0.17$ & $2.68 \pm 1.32$ \\
\hline 103 & 4,8,12,16-Tetramethylheptadecan-4-olide & 2383 & - & $0.33 \pm 0.11$ \\
\hline
\end{tabular}

SD—standard deviation; *-correct isomer is not identified.

The majority of the identified VOCs in both HD-FrCa and HD-DrCa belong to the group of aliphatic compounds-more precisely, saturated hydrocarbons (50.53\% HDFrCa; $27.88 \%$ HD-DrCa), Table 2. The prevalent compound in HD-FrCa was saturated hydrocarbon heptadecane (29.32\%), which was found with 2.4 times lower abundance in HD-DrCa (12.29\%). The origin of heptadecane was connected with direct decarboxylation of stearic acid [19]. The second most abundant saturated hydrocarbon, pentadecane, decreased 1.8 times during air-drying (HD-FrCa 3.25\%; HD-DrCa 1.84\%), and the most abundant unsaturated hydrocarbon, pentadec-1-ene, decreased 3.2 times after air-drying (HD-FrCa 2.12\%; HD-DrCa 0.67\%). Although hexadecane was previously found in C. bursa among the major constituents [12], pentadecane was found with a small percentage only in air-dried C. bursa hydrodistillate. The group of chlorophyll derivatives, as the second most represented group $(22.80 \%)$, and carotenoid degradation products (norisoprenoids) $(7.03 \%)$ showed significant increase in HD-DrCa. The major photosynthetic pigments of green algae are chlorophyll $a$ and $b$ [25]. Phytol, acyclic diterpene alcohol, is linked to the chlorophyll forming ester linkage [26]. The percentage of $(E)$-phytol increased from $5.00 \%$ in $\mathrm{HD}-\mathrm{FrCa}$ to $16.99 \%$ in HD-DrCa, indicating chlorophyll degradation. The percentage of phytone, the oxidation product of phytol, increased from 2.03\% in HD-FrCa to 5.81\% in HD-DrCa. Jerković et al. [12] reported a great increase of phytol and phytone in hydrodistillate of air-dried C. bursa. Five norisoprenoids were identified in HD-FrCa: $C_{11}$-norisoprenoid ( $\beta$-cyclohomocitral), and $\mathrm{C}_{13}$-norisoprenoids (3,4-dehydroionene, hexahydropseudoionone, $\alpha$-ionone and $\beta$-ionene). The oxidative cleavage of carotenoids leads to the formation of norisoprenoid compounds. Due to differences in the length of carotenoid precursor chain and possible positions of the cleavage, $\mathrm{C}_{9}$ - to $\mathrm{C}_{13}$-norisoprenoids could be formed [27]. 
Hence, in HD-DrCa, two $C_{10}$-norisoprenoids (safranal and $\beta$-cyclocitral) and one $\mathrm{C}_{13^{-}}$ norisoprenoid ( $\beta$-ionone) were identified. $\alpha$-Ionone and $\beta$-ionene showed the percentage increase during air-drying (probably due to the oxidation): $\alpha$-ionone $(0.61 \%$ in $\mathrm{HD}-\mathrm{FrCa}$; $5.84 \%$ in $\mathrm{HD}-\mathrm{DrCa}$ ) and $\beta$-ionene (0.85\% in HD-FrCa; $1.81 \%$ in HD-DrCa). Even though hydrodistillation is not very adequate for long chain fatty acids determination (since they are semivolatile or nonvolatile) several fatty acids were detected: dodecanoic (C12:0) acid (0.52\% in HD-FrCa; $0.19 \%$ in HD-DrCa), tetradecanoic (C14:0) acid (2.43\% in HD-FrCa; $0.24 \%$ in HD-DrCa), hexadecanoic (C16:0) acid (8.62\% in HD-FrCa; $10.55 \%$ in HD-DrCa) and (9Z)-octadec-9-enoic (C18:1n9c) acid (0.49\% in HD-FrCa; 2.53\% in HD-DrCa). Distribution of the compound structural groups in HD-FrCa and HD-DrCa is presented in Figure 2.

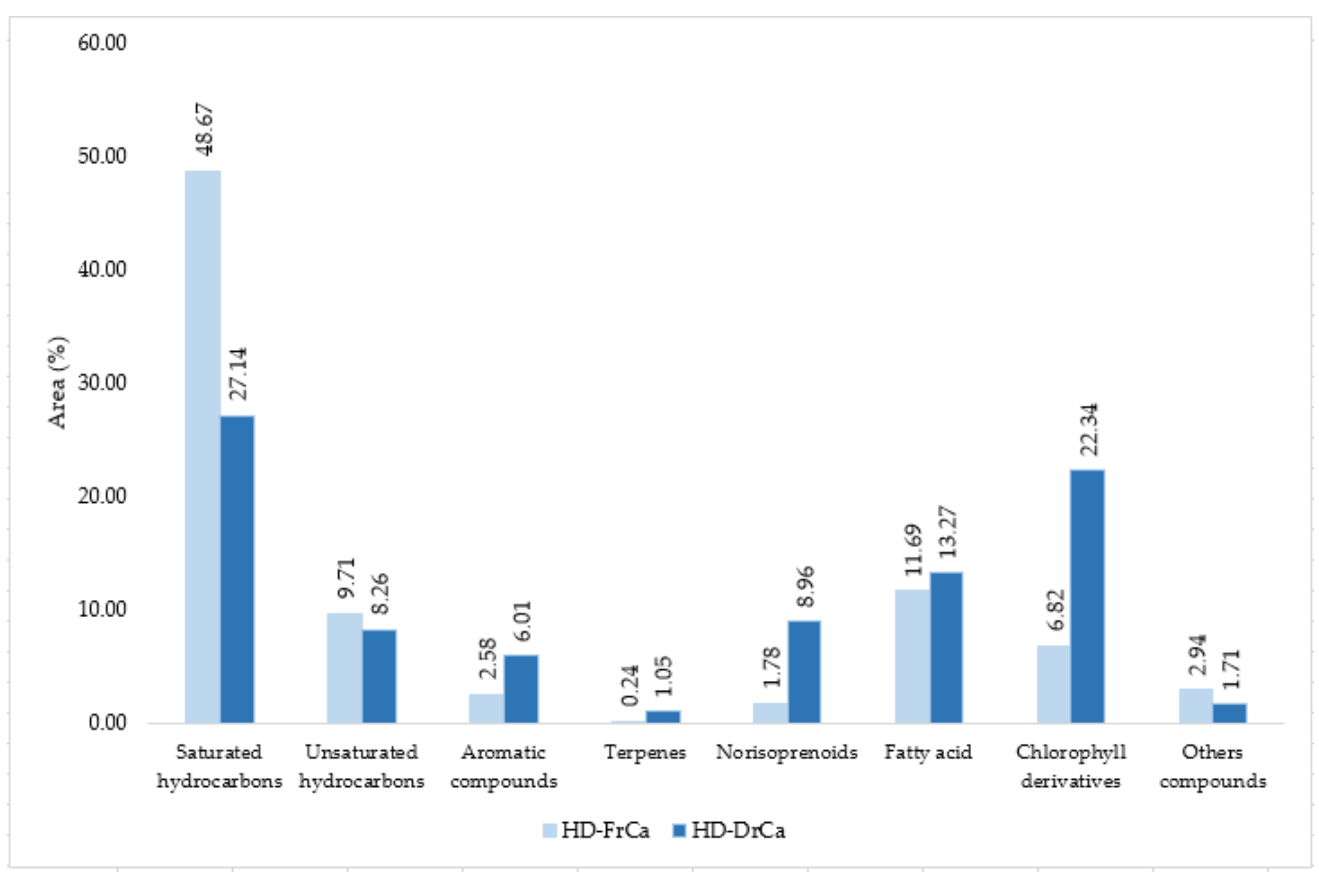

Figure 2. The VOCs from C. adhaerens obtained by HD sorted by structural groups.

\subsection{Fatty Acid Composition}

A total of 13 fatty acids were identified in freeze dried C. adhaerens (FdCa), Table 3. The most abundant fatty acids (FAs) were palmitic (C16:0) and arachidic (C20:0) acids with average values $25.50 \%$ and $22.48 \%$, respectively.

Pereira et al. [28] and Andrade et al. [6] also confirmed the dominance of the mentioned fatty acids in marine green algae from Codium species. Generally, the total saturated fatty acid (SFA) content $(64.94 \%)$ was higher than the content of unsaturated fatty acids (UFAs), which was $35.51 \%$ (24.66\% for monounsaturated FAs (MUFAs) and $10.85 \%$ for polyunsaturated FAs (PUFAs)). The prevalence of SFAs over UFAs in Codium species has been reported previously $[6,28,29]$. Oleic acid isomers $(\mathrm{C} 18: 1 \mathrm{n} 9 c+\mathrm{C} 18: 1 \mathrm{n} 9 t)$ were the main unsaturated fatty acids (16.91\%) followed by palmitoleic acid (C16:1) and cis-linoleic acid (C18:2n6c) whose content was 5.79\% and 4.67\%, respectively. Although SFAs are dominant in analysed C. adhaerens, $\omega 6$ FAs were presented in higher content than $\omega 3$ FAs (Table 3). Higher $\omega 6$ FAs than $\omega 3$ FAs content was found in a previous study [9] for C. bursa. PUFAs are well known for their positive impact on human health. The importance is even higher if the $\omega 6$ FAs: $\omega 3$ FAs ratio in food is between 1.5 and 3 that is generally accepted as the balance value for human nutrition [30]. Considering that the $\omega 6$ FAs: $\omega 3$ FAs ratio obtained in this study was 2.92, marine green alga C. adhaerens can be considered a good source of dietary PUFAs. 
Table 3. Fatty acid composition of C. adhaerens determined by GC-FID.

\begin{tabular}{|c|c|c|}
\hline No. & Fatty Acid & $\mathrm{Av} \pm \mathrm{SD}(\%)$ \\
\hline 1 & Dodecanoic acid (Lauric acid) (C12:0) & $4.02 \pm 1.16$ \\
\hline 2 & Tetradecanoic acid (Myristic acid) (C14:0) & $4.10 \pm 0.28$ \\
\hline 3 & Hexadecanoic acid (Palmitic acid) (C16:0) & $25.50 \pm 0.13$ \\
\hline 4 & Octadecanoic acid (Stearic acid) (C18:0) & $7.12 \pm 0.26$ \\
\hline 5 & Eicosanoic acid (Arachidic acid) (C20:0) & $22.48 \pm 0.49$ \\
\hline \multirow[t]{2}{*}{6} & Docosanoic acid (Behenic acid) (C22:0) & $1.72 \pm 0.19$ \\
\hline & Total saturated fatty acids (SFA) & 64.94 \\
\hline 7 & (9Z)-Hexadec-9-enoic acid (Palmitoleic acid) (C16:1) & $5.79 \pm 0.65$ \\
\hline 8 & (10Z)-Heptadec-10-enoic acid (cis-Heptadecenoic acid) (C17:1) & $1.96 \pm 0.24$ \\
\hline \multirow[t]{2}{*}{9} & $\begin{array}{l}\text { (9Z)-Octadec-9-enoic acid+(9E)-Octadec-9-enoic acid } \\
\text { (cis-Oleic acid+trans-Oleic acid) }(\mathrm{C} 18: \ln 9 c+t)\end{array}$ & $16.91 \pm 1.46$ \\
\hline & Total monounsaturated fatty acids (MUFA) & 24.66 \\
\hline 10 & $\begin{array}{l}\text { (9Z,12Z)-Octadeca-9,12-dienoic acid } \\
\quad(\text { cis-Linoleic acid) }(\mathrm{C} 18: 2 \mathrm{n} 6 \mathrm{c})\end{array}$ & $4.67 \pm 0.33$ \\
\hline 11 & $\begin{array}{c}(9 Z, 12 Z, 15 Z) \text {-Octadeca-9,12,15-trienoic acid } \\
(\alpha \text {-Linolenic acid) }(\mathrm{C} 18: 3 \mathrm{n} 3)\end{array}$ & $2.77 \pm 0.41$ \\
\hline 12 & $\begin{array}{c}(5 Z, 8 Z, 11 Z, 14 Z) \text {-Icosa-5,8,11,14-tetraenoic acid } \\
\text { (Arachidonic acid) (C20:4n6) }\end{array}$ & $1.78 \pm 0.32$ \\
\hline \multirow[t]{4}{*}{13} & $\begin{array}{l}\text { (13Z,16Z)-Docosa-13,16-dienoic acid } \\
\text { (Docosadienoic acid) (C22:2n6) }\end{array}$ & $1.63 \pm 0.49$ \\
\hline & Total polyunsaturated fatty acids (PUFA) & 10.85 \\
\hline & Total $\omega 3$ fatty acids & 2.77 \\
\hline & Total $\omega 6$ fatty acids & 8.08 \\
\hline
\end{tabular}

\subsection{Less Polar Non-Volatile Compounds from F3 and F4 Fractions}

The FdCa sample was fractionated (Section 3.7) to obtain less polar fractions F3 and F4, which were analysed by high-performance liquid chromatography-high-resolution mass spectrometry with electrospray ionisation (UHPLC-ESI(+)-HRMS). The major compounds (in terms of signal intensity) from the obtained chromatograms in positive ion mode were tentatively identified on the basis of their elemental compositions and tandem mass spectra (Table 4), and they belong to: chlorophyll derivatives, fatty acid glycerides and related compounds, terpenes, steroids, and carotenoids. Total ion chromatograms of the fractions F3 and F4 are shown in Figure 3 and extracted ion chromatograms (XIC) of most abundant ions in the fractions F3 and F4 are shown in Figure 4.

Table 4. Major non-volatile compounds in F3 and F4 fractions and their tentative identification by UHPLC-ESI(+)-HRMS.

\begin{tabular}{|c|c|c|c|c|c|c|}
\hline \multirow[t]{2}{*}{ No. } & \multirow[t]{2}{*}{ Compound } & \multirow{2}{*}{ Rt (min) } & \multirow{2}{*}{$\begin{array}{l}\text { Elemental } \\
\text { Composition }\end{array}$} & \multirow{2}{*}{$\begin{array}{l}m / z \\
\text { (Error, ppm) }\end{array}$} & \multicolumn{2}{|c|}{$\begin{array}{c}\text { Peak Area } \\
\text { (Arbitrary Units) }\end{array}$} \\
\hline & & & & & F3 & F4 \\
\hline 1 & Gingerglycolipid A & 11.194 & $\mathrm{C}_{33} \mathrm{H}_{56} \mathrm{O}_{14}$ & $\begin{array}{l}677.37267 \\
(2.379)\end{array}$ & $276,841.59$ & 1288.25 \\
\hline 2 & $\begin{array}{c}\text { 2-Hydroxy-3-( } \beta \text {-L-talopyranosyloxy)propyl } \\
(9 Z, 12 Z, 15 Z) \text {-octadeca-9,12,15-trienoate }\end{array}$ & 11.976 & $\mathrm{C}_{27} \mathrm{H}_{46} \mathrm{O}_{9}$ & $\begin{array}{l}515.32020 \\
(2.445)\end{array}$ & $469,972.09$ & 3546.75 \\
\hline 3 & $\begin{array}{l}\text { 1,3-Dihydroxy-2-propanyl } \\
\text { icosa-5,8,11,14-tetraenoate }\end{array}$ & 13.971 & $\mathrm{C}_{23} \mathrm{H}_{38} \mathrm{O}_{4}$ & $\begin{array}{l}379.28354 \\
(1.972)\end{array}$ & $54,160.86$ & $28,397.43$ \\
\hline
\end{tabular}


Table 4. Cont.

\begin{tabular}{|c|c|c|c|c|c|c|}
\hline \multirow{2}{*}{ No. } & \multirow{2}{*}{ Compound } & \multirow{2}{*}{ Rt (min) } & \multirow{2}{*}{$\begin{array}{l}\text { Elemental } \\
\text { Composition }\end{array}$} & \multirow{2}{*}{$\begin{array}{l}m / z \\
\text { (Error, ppm) }\end{array}$} & \multicolumn{2}{|c|}{$\begin{array}{c}\text { Peak Area } \\
\text { (Arbitrary Units) }\end{array}$} \\
\hline & & & & & F3 & F4 \\
\hline 4 & 2,3-Dihydroxypropyl palmitate & 14.222 & $\mathrm{C}_{19} \mathrm{H}_{38} \mathrm{O}_{4}$ & $\begin{array}{l}331.28296 \\
(4.030)\end{array}$ & $377,335.75$ & $495,766.09$ \\
\hline 5 & 2,3-Dihydroxypropyl octadec-9-enoate & 14.561 & $\mathrm{C}_{21} \mathrm{H}_{40} \mathrm{O}_{4}$ & $\begin{array}{c}357.30074 \\
(-2.247)\end{array}$ & $40,879.4$ & - \\
\hline 6 & Isoamijiol oxidation product * & 14.939 & $\mathrm{C}_{20} \mathrm{H}_{30} \mathrm{O}_{2}$ & $\begin{array}{l}303.23271 \\
(-2.803)\end{array}$ & $994,098.63$ & $18,848.2$ \\
\hline 7 & 2,3-Dihydroxypropyl stearate & 15.254 & $\mathrm{C}_{21} \mathrm{H}_{42} \mathrm{O}_{4}$ & $\begin{array}{l}359.31454 \\
(2.932)\end{array}$ & $505,462.75$ & $659,353.94$ \\
\hline 8 & 2-Hydroxypropyl palmitate & 15.379 & $\mathrm{C}_{19} \mathrm{H}_{38} \mathrm{O}_{3}$ & $\begin{array}{c}315.29087 \\
(-4.761)\end{array}$ & $52,074.77$ & $141,670.42$ \\
\hline 9 & Pheophorbide $a$ & 15.537 & $\mathrm{C}_{35} \mathrm{H}_{36} \mathrm{~N}_{4} \mathrm{O}_{5}$ & $\begin{array}{c}593.27486 \\
(1.663)\end{array}$ & $1,478,183.5$ & $175,460.75$ \\
\hline 10 & Isoamijiol & 15.569 & $\mathrm{C}_{20} \mathrm{H}_{32} \mathrm{O}_{2}$ & $\begin{array}{l}305.24830 \\
(-2.598)\end{array}$ & $552,684.31$ & $66,746.24$ \\
\hline 11 & Fucoxanthin & 15.6 & $\mathrm{C}_{42} \mathrm{H}_{58} \mathrm{O}_{6}$ & $\begin{array}{c}659.42919 \\
(2.169)\end{array}$ & $23,608.59$ & $20,924.63$ \\
\hline 12 & $\begin{array}{c}\text { (2E)-3-[21-(Methoxycarbonyl)-4,8,13,18- } \\
\text { tetramethyl-20-oxo-9,14-divinyl-3,4- } \\
\text { didehydro-3-24,25-dihydrophorbinyl]acrylic } \\
\text { acid }\end{array}$ & 15.632 & $\mathrm{C}_{35} \mathrm{H}_{30} \mathrm{~N}_{4} \mathrm{O}_{5}$ & $\begin{array}{l}587.22619 \\
\quad(4.618)\end{array}$ & $16,648.76$ & $94,242.22$ \\
\hline 13 & $\begin{array}{c}\text { 3-[21-(Methoxycarbonyl)-4,8,13,18- } \\
\text { tetramethyl-20-oxo-9,14-divinyl-3,4- } \\
\text { didehydro-3-24,25- } \\
\text { dihydrophorbinyl]propanoic } \\
\text { acid }\end{array}$ & 15.632 & $\mathrm{C}_{35} \mathrm{H}_{32} \mathrm{~N}_{4} \mathrm{O}_{5}$ & $\begin{array}{l}589.24226 \\
(3.887)\end{array}$ & $18,364.77$ & $62,398.75$ \\
\hline 14 & 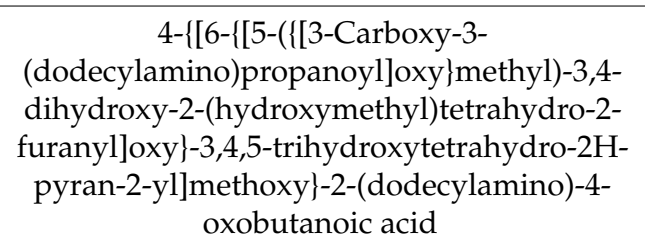 & 15.632 & $\mathrm{C}_{44} \mathrm{H}_{80} \mathrm{~N}_{2} \mathrm{O}_{17}$ & $\begin{array}{c}909.55133 \\
(1.815)\end{array}$ & $387,109.44$ & 3907.02 \\
\hline 15 & 13-Docosenamide & 16.103 & $\mathrm{C}_{22} \mathrm{H}_{43} \mathrm{NO}$ & $\begin{array}{c}338.34153 \\
(0.637)\end{array}$ & 3958,621 & $859,522.19$ \\
\hline 16 & 2-Hydroxypropyl stearate & 16.29 & $\mathrm{C}_{21} \mathrm{H}_{42} \mathrm{O}_{3}$ & $\begin{array}{l}343.32107 \\
(-1.159)\end{array}$ & $114,486.16$ & $343,931.75$ \\
\hline 17 & $\begin{array}{c}\text { 3-(ß-D-Galactopyranosyloxy)-2- } \\
\text { [(7Z,10Z,13Z)-7,10,13- } \\
\text { hexadecatrienoyloxy]propyl } \\
(9 Z, 12 Z, 15 Z) \text {-octadeca-9,12,15-trienoate }\end{array}$ & 16.605 & $\mathrm{C}_{43} \mathrm{H}_{70} \mathrm{O}_{10}$ & $\begin{array}{l}747.50298 \\
(1.590)\end{array}$ & $755,057.44$ & $17,590.1$ \\
\hline 18 & $\begin{array}{l}(3 \beta, 6 \alpha) \text {-14-Methylergosta-8,24(28)-diene-3,6- } \\
\text { diol }\end{array}$ & 17.512 & $\mathrm{C}_{29} \mathrm{H}_{48} \mathrm{O}_{2}$ & $\begin{array}{l}429.37200 \\
(1.646)\end{array}$ & $1,082,618.75$ & $58,710.11$ \\
\hline 19 & $\beta$-Stigmasterol & 17.701 & $\mathrm{C}_{29} \mathrm{H}_{46}$ & $\begin{array}{l}395.36791 * * \\
(-1.717)\end{array}$ & $58,667.22$ & $514,958.41$ \\
\hline 20 & $\begin{array}{l}\text { 1-Hydroxy-3-(tetradecanoyloxy)-2-propanyl } \\
\text { (9Z)-9-octadecenoate }\end{array}$ & 17.985 & $\mathrm{C}_{35} \mathrm{H}_{66} \mathrm{O}_{5}$ & $\begin{array}{c}567.49797 \\
(0.580)\end{array}$ & $28,771.63$ & $419,526.25$ \\
\hline
\end{tabular}


Table 4. Cont

\begin{tabular}{|c|c|c|c|c|c|c|}
\hline \multirow[t]{2}{*}{ No. } & \multirow{2}{*}{ Compound } & \multirow{2}{*}{ Rt (min) } & \multirow{2}{*}{$\begin{array}{l}\text { Elemental } \\
\text { Composition }\end{array}$} & \multirow{2}{*}{$\begin{array}{c}m / z \\
\text { (Error, ppm) }\end{array}$} & \multicolumn{2}{|c|}{$\begin{array}{c}\text { Peak Area } \\
\text { (Arbitrary Units) }\end{array}$} \\
\hline & & & & & F3 & F4 \\
\hline 21 & (3ß)-3-Hydroxystigmast-5-en-7-one & 18.364 & $\mathrm{C}_{29} \mathrm{H}_{48} \mathrm{O}_{2}$ & $\begin{array}{l}429.37170 \\
(2.352)\end{array}$ & 440,882 & $287,291.56$ \\
\hline 22 & 3-Hydroxy-1,2-propanediyl bis(9-octadecenoate) & 19.631 & $\mathrm{C}_{39} \mathrm{H}_{72} \mathrm{O}_{5}$ & $\begin{array}{c}621.54313 \\
(3.412)\end{array}$ & $76,716.06$ & $96,810.66$ \\
\hline 23 & 3-Hydroxy-2-(palmitoyloxy)propyl stearate & 19.663 & $\mathrm{C}_{37} \mathrm{H}_{72} \mathrm{O}_{5}$ & $\begin{array}{l}597.54727 \\
(-3.374)\end{array}$ & $134,634.83$ & $139,876.53$ \\
\hline 24 & $\begin{array}{c}\text { Methyl } \\
\text { 14-ethyl-4,8,13,18-tetramethyl-20-oxo-3-(3-oxo-3- } \\
\{[(2 E)-3,7,11,15 \text {-tetramethyl-2-hexadecen-1- } \\
\text { yl]oxy\}propyl)-9-vinyl-3,4-didehydro-24,25- } \\
\text { dihydrophorbine-21-carboxylate }\end{array}$ & 19.982 & $\mathrm{C}_{55} \mathrm{H}_{72} \mathrm{~N}_{4} \mathrm{O}_{5}$ & $\begin{array}{c}869.55660 \\
(1.098)\end{array}$ & - & $281,383.5$ \\
\hline 25 & $\begin{array}{l}\text { Methyl 9-acetyl-14-ethylidene-4,8,13,18- } \\
\text { tetramethyl-20-oxo-3-\{3-oxo-3-[(3,7,11,15- } \\
\text { tetramethyl-2-hexadecen-1-yl)oxy]propyl\}-13,14- } \\
\text { dihydro-21-phorbinecarboxylate }\end{array}$ & 20.011 & $\mathrm{C}_{55} \mathrm{H}_{74} \mathrm{~N}_{4} \mathrm{O}_{6}$ & $\begin{array}{c}887.57206 \\
(-4.454)\end{array}$ & $33,461.45$ & $18,710,810$ \\
\hline 26 & $\begin{array}{c}\text { Methyl (10Z,14Z,20Z)-12-ethyl-3-hydroxy- } \\
\text { 13,18,22,27-tetramethyl-5-oxo-23-(3-oxo-3- }\{[(2 E)- \\
\text { 3,7,11,15-tetramethyl-2-hexadecen-1- } \\
\text { yl]oxy\}propyl)-17-vinyl-4-oxa-8,24,25,26- } \\
\left.\text { tetraazahexacyclo[19.2.1.16,9.11,14.16,19.0.7 } .0^{2,7}\right] \text { hep- } \\
\text { tacosa-1(24),2(7),6(27),8,10,12,14,16,18,20- } \\
\text { decaene-3-carboxylate }\end{array}$ & 20.013 & $\mathrm{C}_{55} \mathrm{H}_{74} \mathrm{~N}_{4} \mathrm{O}_{7}$ & $\begin{array}{c}903.56436 \\
(-1.473)\end{array}$ & - & 4582,960 \\
\hline 27 & Pheophytin $a$ & 20.168 & $\mathrm{C}_{55} \mathrm{H}_{74} \mathrm{~N}_{4} \mathrm{O}_{5}$ & $\begin{array}{c}871.57287 \\
(0.375)\end{array}$ & - & $45,562,208$ \\
\hline
\end{tabular}

*-exact compound not determined; ${ }^{* *}$-dehydrated molecule $\left[\mathrm{M}-\mathrm{H}_{2} \mathrm{O}+\mathrm{H}\right]^{+}$.

(a)

THG (APV+, CE $=10.0 \mathrm{eV}$ )

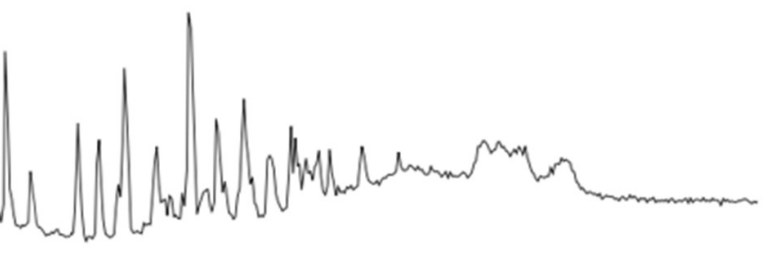

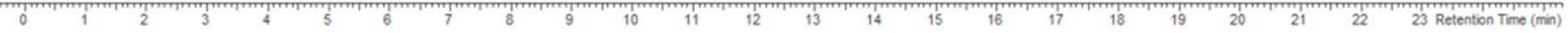

(b)

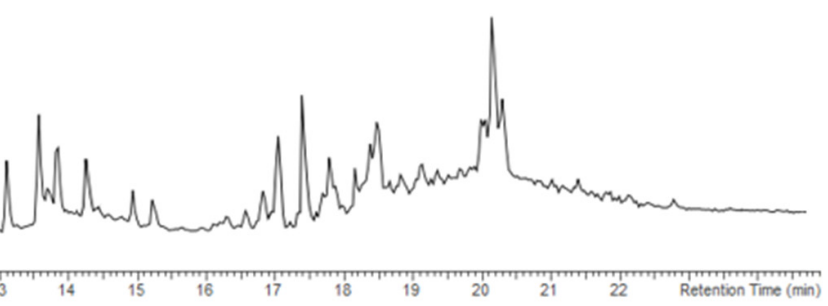

TIC $(\mathrm{API}+, \mathrm{CE}=10.0 \mathrm{eV}$

Figure 3. Total ion chromatogram (TIC) of the fractions (a) F3 and (b) F4. 


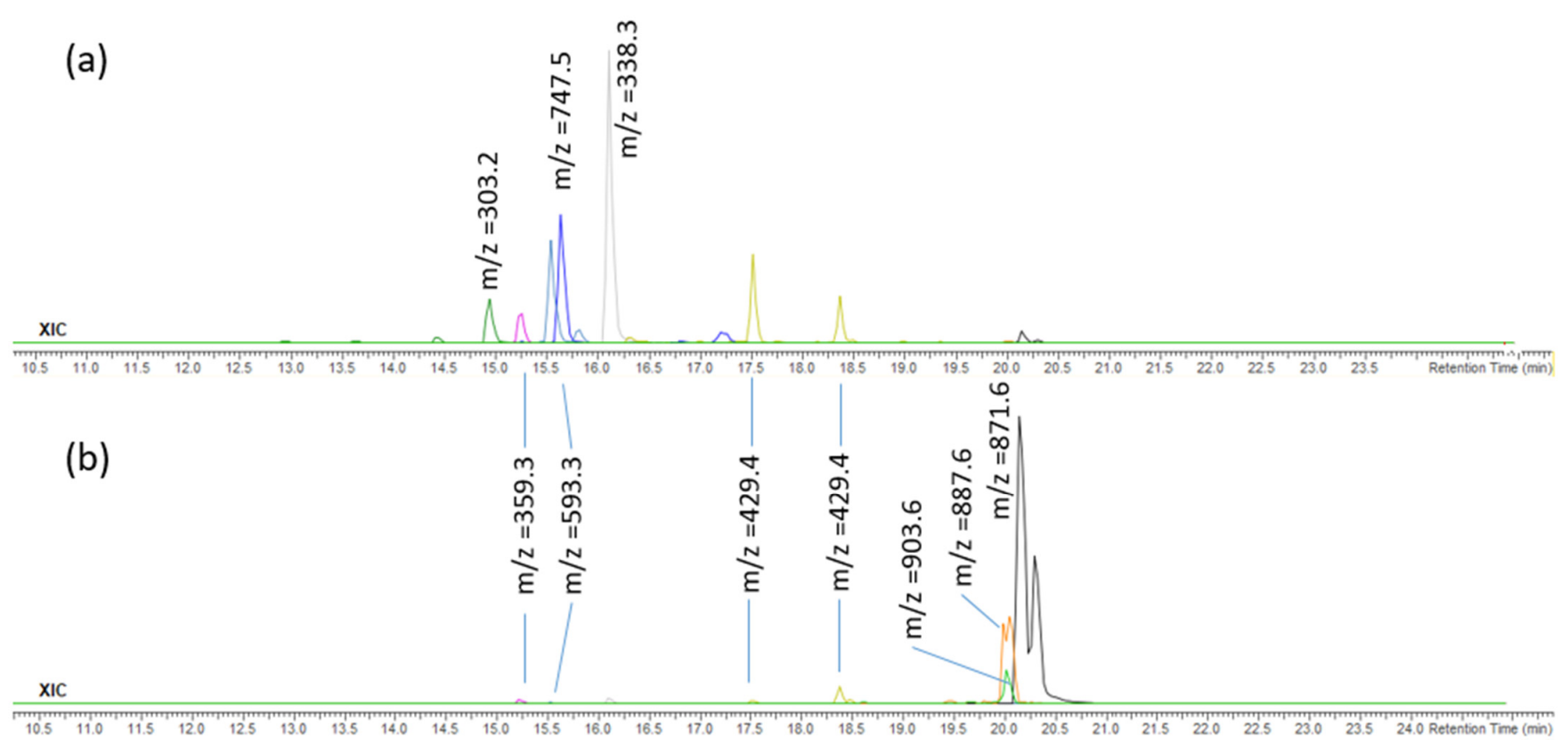

Figure 4. Extracted ion chromatograms (XIC) of most abundant ions in the fractions (a) F3 and (b) F4 (zoomed).

Well-known green pigment chlorophyll was not detected in the fractions, but its derivatives devoid of magnesium atoms constituted the major compounds, particularly in F4 (Table 4). Identified chlorophyll derivatives can be divided into two sub-groups containing 55 or 35 (without the aliphatic side chain) carbon atoms. The first subgroup includes four highly lipophilic compounds with significant abundance mainly in F4, except for the compound 18 (Table 4), which was also present in F3, since it is more polar due to the additional keto group. The main component of this sub-group is pheophytin $a$, which has previously been found in notable amounts in different macroalgae [31]. Pheophytin $a$ has been identified from edible green alga Enteromorpha prolifera, and showed a potent anti-inflammatory activity [32], and has been reported to be a potent suppressive substance against $и т и \mathrm{C}$ gene expression in a tester bacteria induced by genotoxic substances [33]. In addition, previous studies have reported that pheophytin $a$ and pheophytin $a$-related compounds show antioxidant activity in the autooxidation of lipids [34,35]. The other three compounds of this subgroup were pheophytin $a$ derivatives characterised by the presence of an additional double bond, carbonyl, or hydroxyl group in their composition. The second subgroup consisted of three compounds (Table 4) having the common structure (pheophorbide $a$ and its derivatives differ from pheophytin $a$ by the absence of the long side hydrocarbon chain). Pheophorbide $a$ was much more abundant in F3 than in F4. It is a photosensitiser that can induce significant anti-proliferative effects in several human cancer cell lines [36].

Fatty acid glycerides in F3 and F4 consisted of monoglycerides of palmitic, stearic, oleic, and arachidonic acids that were found prevailing in C. adhaerens fatty acid composition (Table 3). Three diglycerides (one of stearic acid and two of oleic acid) were found (Table 4). Three other compounds, chemically related to this group, were identified as fatty amide (13-docosenamide), fatty acid esters (palmitate and stearate) of 2-hydroxypropanol. Sugar fatty acid ester (octadecatrienoate) and diester of octadecatrienoic and hexadecatrienoic acids were also found. One glycosylmonoacylglycerol (gingerglycolipid A) was present. In addition, the high molecular derivative of 4-oxobutanoic acid with elemental composition $\mathrm{C}_{44} \mathrm{H}_{80} \mathrm{~N}_{2} \mathrm{O}_{17}$ was identified (Table 4, compound 14).

Terpenes and steroids comprised five compounds (Table 4). The tandem mass spectrum of $\mathrm{C}_{20} \mathrm{H}_{32} \mathrm{O}_{2}$ showed two $\mathrm{H}_{2} \mathrm{O}$ molecules loss and specific carbon backbone fragmentation pattern that permitted assigning diterpenoid isoamijiol structure, similar to $F$. virsoides [8]. Previously, it was isolated from brown alga Dictyota linearis [37]. Another 
compound (more abundant in F3) with a similar retention time and the same molecular formula $\mathrm{C}_{20} \mathrm{H}_{30} \mathrm{O}_{2}$ containing only one hydroxyl was tentatively identified as isoamijiol oxidation product possessing keto-group. Two major steroids (Table 4 ) were identified as monool ( $\beta$-stigmasterol) and (3 $\beta$ )-3-hydroxystigmast-5-en-7-one possessing hydroxy and keto groups.

Among xanthophyll carotenoids, only fucoxanthin was found in F3 and F4. It has been reported as the main carotenoid pigment in all brown algae $[11,38]$ that possess different biological activities, i.e., antioxidant and anticancer $[39,40]$.

\subsection{Antioxidant Activity of F3 and F4 Fractions In Vitro}

Within this study, in vitro evaluation of the antioxidant activity of two less polar fractions F3 and F4 obtained from C. adhaerens was performed using four different spectroscopic methods: Folin-Ciocalteu (F-C), FRAP, DPPH, and ABTS assays. Although F-C assay is commonly known as a measure of total phenolic content, here it represents a rate of an overall antioxidant activity since the extracts do not contain phenols (as in our previous study on F. virsoides [11]). The results obtained using F-C assay indicated 3-fold higher $(p<0.0001)$ activity of F4 $(64.27 \pm 0.73 \mathrm{mg} \mathrm{GAE} / \mathrm{g}$ F4) then F3 $(21.12 \pm 0.91 \mathrm{mg}$ GAE /g F3). Interestingly, although the obtained values correspond to the values from our two previously published studies on Amphiroa rigida [41] and Fucus virsoides [11], one peculiarity can be observed. In C. adhaerens, higher activity was observed for F4, which is not the case in the other two macroalgae where higher activity is obtained in F3. This aberration could be explained by the different chemical compositions of $C$. adhaerens fractions (Table 4). The antioxidant activity of F3 and F4 was further tested by implementing additional two antioxidant assays-DPPH and FRAP, as depicted in Figure 5. Although DPPH implies dominant reaction through single electron transfer (SET), DPPH radical can also react through transfer of hydrogen atom (HAT), while FRAP assay is only based on SET. The inhibition percentage using DPPH assay for a tested concentration of $1 \mathrm{mg} / \mathrm{mL}$ for both fractions was around 20\%. Nevertheless, when normalised per gram of the fraction, F3 (59.69 $\pm 2.32 \mathrm{mg}$ AAE/g fraction) showed slightly higher $(p<0.005)$ antioxidant activity then F4 (44.34 $\pm 4.05 \mathrm{mg} \mathrm{AAE} / \mathrm{g}$ fraction). However, the reverse result was obtained when conducting FRAP analysis. Higher activity $(p<0.0001)$ was obtained for F4 (6.54 $\pm 0.15 \mathrm{mmol}$ ferrous eq./g fraction), then F3 (4.80 $\pm 0.04 \mathrm{mmol}$ ferrous eq. $/ \mathrm{g}$ fraction). In F4, the dominant compound is pheophytin $a$ along with its derivatives which are known for their antioxidant ability [42], while in F3 pheophytin $a$ is not detected. This is in accordance with the results obtained using FRAP assay, where higher activity was observed in F4, thus indicating easier reaction through single electron transfer probably due to pheophytin $a$ and its side chain in the molecular structure. Conversely, the results obtained using DPPH assay showed relatively low antioxidant activity of both fractions, but slightly higher activity for F3 could be explained by the presence of pheophorbide $a(\mathrm{PPB} a)$, which is also a known product related to the chlorophyll with already proven biological activity [36]. Sudha et al. [43] evaluated the antioxidant activity of C. adhaerens by implementing soxhlet extraction with ethyl acetate as solvent. The obtained extract showed the highest activity against free DPPH radical at the concentration of $1000 \mu \mathrm{g} / \mathrm{mL}$ with an inhibition percentage of around $70 \%$.

Additionally, the reduction of radical cation by implementing ABTS assay was also evaluated. To obtain the $\mathrm{IC}_{50}$ curve, different concentrations of $\mathrm{F} 3$ and $\mathrm{F} 4$ were prepared ranging from 0.05 to $5 \mathrm{mg} / \mathrm{mL}$, followed by increasing the inhibition percentage. $\mathrm{IC}_{50}$ values for both F3 and F4 were calculated as shown in Table 5 with the corresponding confidence interval, slope and coefficient of determination $\left(\mathrm{R}^{2}\right)$. As can be seen, the $\mathrm{IC}_{50}$ value for both fractions is almost identical and is around $2.4 \mathrm{mg} / \mathrm{mL}$, which represents the higher concentration responsible for the inhibition of $50 \%$ of free radicals. This could be explained by the dominant presence of different pigments, i.e., the presence of PPB $a$ in F3 and pheophytin $a$ in F4 with the addition of carotenoid fucoxanthin that exhibits antioxidant activity [44] and is present in both fractions in a similar ratio. Pinteus et al. [45] 
also analysed the antioxidant activity of numerous algae, including C. adhaerens, but with an emphasis on more polar compounds. They also observed relatively low antioxidant activity with $\mathrm{IC}_{50}$ values for both methanolic and dichloromethane extracts higher than $1000 \mu \mathrm{g} / \mathrm{mL}$ [45]. The inhibition percentage using ABTS assay for tested concentration of $1 \mathrm{mg} / \mathrm{mL}$ for both fractions was around $40 \%$ (data not shown).

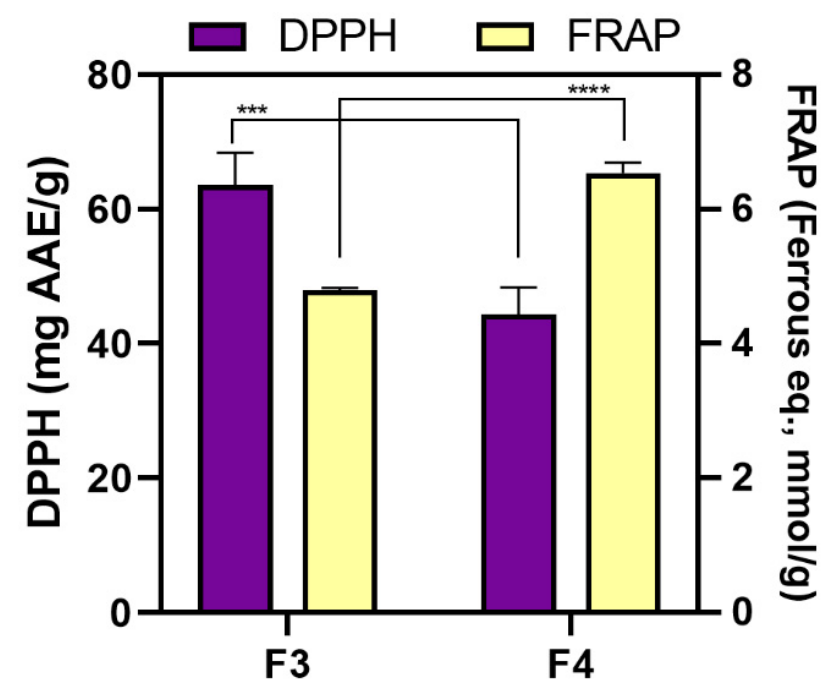

Figure 5. Antioxidant activity of $C$. adhaerens less polar fractions (F3 and F4) obtained using 2,2diphenyl-1-picryl-hydrazyl-hydrate (DPPH) and ferric reducing antioxidant power (FRAP) in vitro assays (mean $\pm \mathrm{SD} ; n=3$ ). An asterisk indicates a significant difference between F3 and F4 $\left({ }^{* * *} p<0.01 ;{ }^{* * * *} p<0.001\right)$.

Table 5. Antioxidant activity of $C$. adhaerens fractions (F3 and F4) obtained using ABTS assay with corresponding $\mathrm{IC}_{50}$ values and the significance parameters (confidence interval, slope and coefficient of determination, $\mathrm{R}^{2}$ ).

\begin{tabular}{ccccc}
\hline Sample & IC $_{\mathbf{5 0}}$ Value, $\mathbf{~ m g} / \mathbf{m L}$ & Confidence Interval & Slope & $\mathbf{R}^{\mathbf{2}}$ \\
\hline F3 & 2.44 & $1.94-3.64$ & 1.36 & 0.997 \\
F4 & 2.49 & $1.92-3.86$ & 1.26 & 0.997 \\
\hline
\end{tabular}

\subsection{Developmental Toxicity of F3 and F4 Fractions in Zebrafish Embryo}

To evaluate the potential toxicity of tested C. adhaerens fractions on embryonic development of zebrafish, mortality, morphological changes and hatchability at 96 hpf were analysed. Upon exposure to 500, 250 and $125 \mu \mathrm{g} / \mathrm{mL}$ of F3 and F4, no statistically significant changes in survival were observed ( $p<0.05$; data not shown). Additionally, no significant morphological abnormalities or hatching inhibition were seen in the tested concentration range. Mortality in the control groups (negative control (AW) and solvent controls ( $1 \% \mathrm{MeOH}, 1 \% \mathrm{DMSO})$ ) was $<5 \%$. Given the obtained results, concentrations of 500,250 and $125 \mu \mathrm{g} / \mathrm{mL}$ were selected for further experiments.

\subsection{Protective Effects of F3 and F4 Fractions against $\mathrm{H}_{2} \mathrm{O}_{2}$-Induced Oxidative Stress}

Although valuable, in vitro methods do not reflect physiological processes like absorption and metabolism of antioxidants, and therefore a compound that shows good antioxidant properties within in vitro tests may not necessarily be biologically active. For that reason, zebrafish Danio rerio embryos were employed for this research, as currently one of the most perspective vertebrate model organisms [46]. To determine whether C. adhaerens fractions play a role in ROS-mediated oxidative stress, zebrafish embryos were exposed to $\mathrm{F} 3$ and $\mathrm{F} 4$ fractions in the presence of $\mathrm{H}_{2} \mathrm{O}_{2}$. A statistically significant increase in the mortality rate was observed in the $\mathrm{H}_{2} \mathrm{O}_{2}$-treated group (66.7 $\pm 5.8 \%$ ). However, 
pre-treatment with the highest tested concentration $(500 \mu \mathrm{g} / \mathrm{mL})$ of both, F3 and F4, declined mortality rates by $23.3 \%$ and $26.7 \%$, respectively, compared to $\mathrm{H}_{2} \mathrm{O}_{2}$-treated group. Lower concentrations $(125 \mu \mathrm{g} / \mathrm{mL}$ and $250 \mu \mathrm{g} / \mathrm{mL})$ of both fractions had no impact on zebrafish survival.

ROS production was significantly elevated by $\mathrm{H}_{2} \mathrm{O}_{2}$ treatment $(255.9 \%)$ when compared to control groups (normalised to $100 \%$ ). A concentration-dependent reduction in the DCF fluorescence intensity was observed in the specimens preincubated with the highest concentration of the fractions (Figure 6). The treatment with $500 \mu \mathrm{g} / \mathrm{mL}$ of F3 and F4 resulted in significant decrease of ROS generation to $154.8 \%(p<0.001)$ and $132.7 \%$ $(p<0.001)$, respectively.
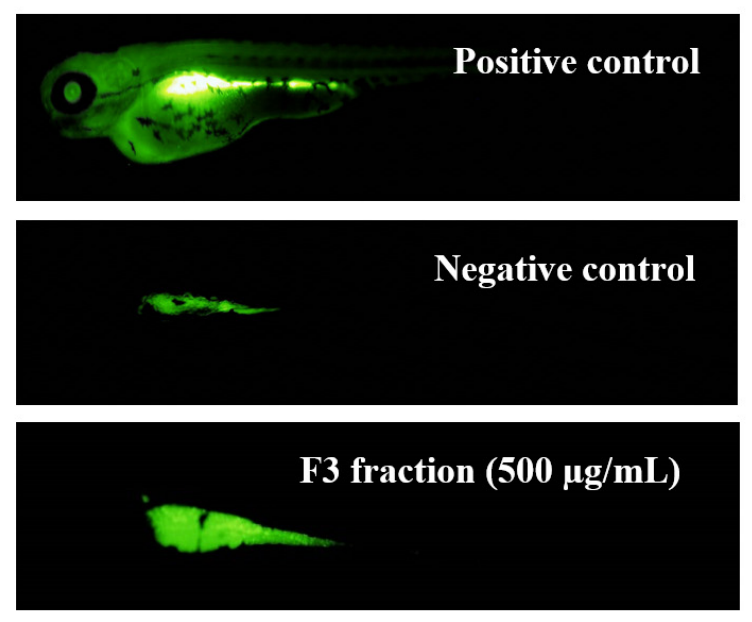

F4 fraction $(500 \mu \mathrm{g} / \mathrm{mL})$
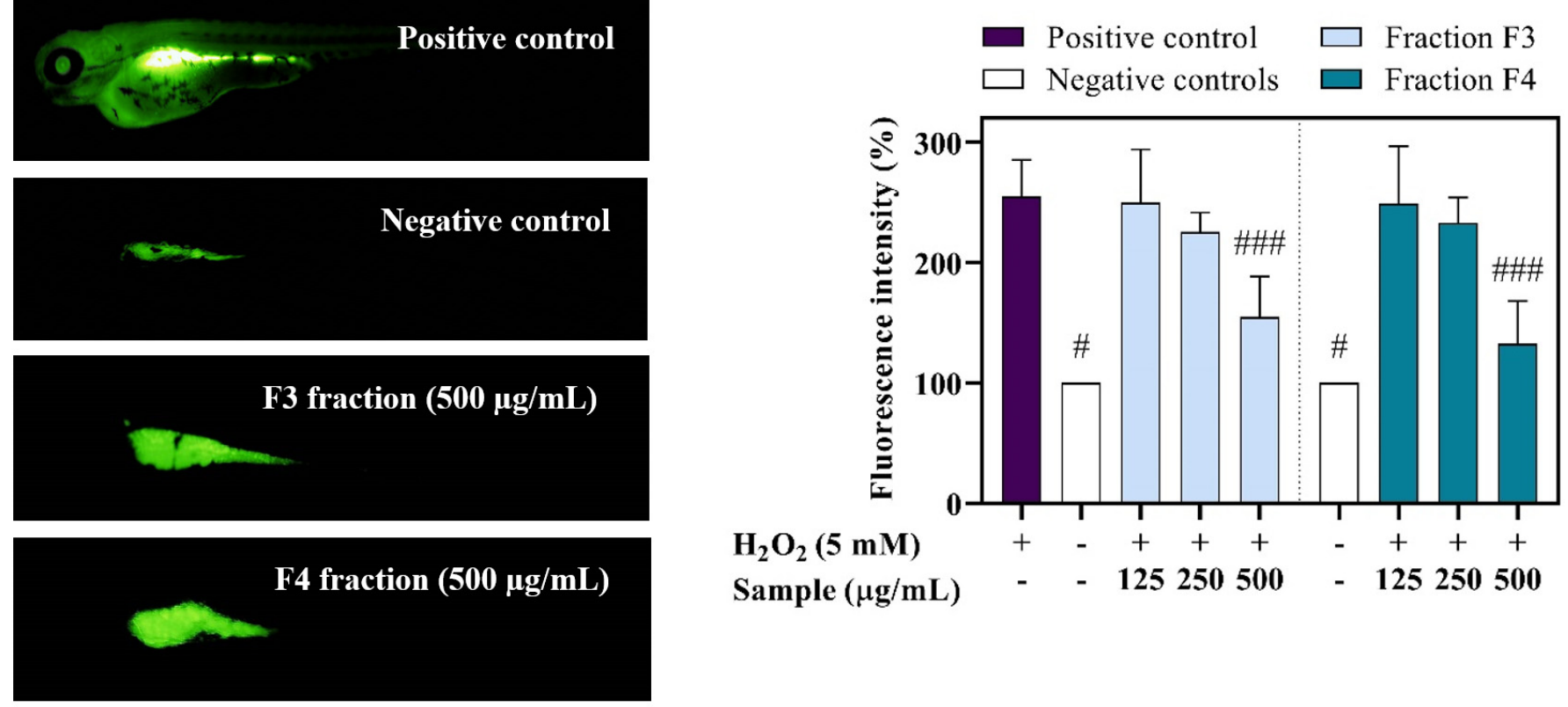

Figure 6. ROS scavenging potential of C. adhaerens fractions F3 and F4 in zebrafish. Left: Representative fluorescence images of $\mathrm{H}_{2} \mathrm{O}_{2}$ treated and the fractions co-treated zebrafish larvae. Right: Mean fluorescent intensity of DCF in the whole larvae calculated using Image J program. Sign \# represents significant difference when compared with $\mathrm{H}_{2} \mathrm{O}_{2}$-treated group (\# $p<0.05 ; \# \#$; $<0.001)$. Each treatment was normalised relative to non-treated controls $(100 \%)$.

Such findings imply that tested fractions protect cells against $\mathrm{H}_{2} \mathrm{O}_{2}$-induced oxidative stress, ultimately leading to a decrease in zebrafish mortality. When compared, the results obtained using the zebrafish in vivo model correlate well with the ones obtained with in vitro methods. According to both used methods, F3 and F4 exerted similar antioxidative responses which is in agreement with the high abundance of the biomolecules in the fractions with already proven antioxidant properties. Specifically, fucoxanthin, pheophytin $a$, and pheophorbide $a$ have attracted extensive interest due to their beneficial biological activities including anti-cancer, anti-inflammatory, anti-oxidant, anti-angiogenic, and antiwrinkle activity [47-49]. Kang et al. [44] demonstrated the protective effects of fucoxanthin (25-100 $\mu \mathrm{M})$ against high glucose-induced oxidative damage in a zebrafish model. Even though antioxidant activities of phaeophytin $a$ and pheophorbide $a$ were not determined using zebrafish model, in vitro methods (DPPH, TBARS assay) proved their strong antioxidant potential [48]. Moreover, the reduction of free radicals and the protection of cultured lymphocytes against oxidative DNA damage was observed in the presence of those two chlorophyll $a$ derivatives $[48,50]$. For that reason, we assume that the high abundance of pheophytin $a$ in F4 contributed to the higher antioxidant potential.

When compared with the results published in our previous study on Fucus virsoides F3 and F4 fractions [8], the antioxidant potential of C. adhaerens was notably lower. For that reason, one should note that the antioxidant activity of each tested fraction cannot be 
related to the particular compound due to possible synergistic/antagonistic interactions between bioactive compounds. Thus, we strongly encourage further studies to revealthe mechanisms behind the functionality of antioxidant mixtures that can ultimately result in implementation in the food, cosmeceutical, and pharmaceutical industry.

\section{Materials and Methods}

\subsection{Chemicals}

The fatty acids methyl esters (FAMEs) were purchased from Supelco Co. (Bellefonte, PA, USA). The standards of L-ascorbic acid $(\geq 99 \%)$, gallic acid $(>97.5 \%)$, DPPH $(2,2-$ diphenyl-1-picrylhydrazyl), TPTZ (2,4,6-tripyridyl-S-triazine, $\geq 98 \%$ ), ABTS (diammonium salt of 2,2'-azino-bis(3-ethylbenzthiazolin-6-yl)sulfonic acid, $>99.0 \%$ ), and dichloro-dihydrofluorescein diacetate ( $\geq 97 \%$, DCF-DA) were purchased from Sigma-Aldrich (St. Louis, MO, USA).

Organic solvents (dimethyl sulfoxide (DMSO, p.a.), ethanol (p.a.), methanol (p.a.)), hydrochloric acid ( $\mathrm{HCl}$, p.a.), iron (III) chloride $\left(\mathrm{FeCl}_{3}\right.$, p.a.), Folin-Ciocalteu reagent and $\mathrm{NaHCO}_{3}$ (p.a.) were obtained from Kemika (Zagreb, Croatia), while potassium persulfate $(>98 \%)$ was purchased from Scharlab (Barcelona, Spain). Hydrogen peroxide $\left(\mathrm{H}_{2} \mathrm{O}_{2}, 30 \%\right)$ was obtained from Alkaloid Skopje (Skopje, North Macedonia).

Acetonitrile with $0.1 \%(v / v)$ formic acid and water with $0.1 \%(v / v)$ formic acid, both hypergrade for HPLC-MS LiChrosolv ${ }^{\circledR}$, were purchased from Supelco Co. (Bellefonte, PA, USA).

Zebrafish D. rerio adults of wild-type WIK strain were obtained from the European Zebrafish Resource Center of the Karlsruhe Institute of Technology (KIT), Karlsruhe, Germany. Used solvents were of HPLC grade and were obtained from J.T. Baker (Bridgewater, NJ, USA).

\subsection{Macroalga Sample}

Codium adhaerens C. Agardh 1822 was collected in November 2020 by a single-point collection from the Adriatic Sea (Poluotok Rtina/Paška vrata) with the sampling geographical coordinates $44^{\circ} 19^{\prime} 14^{\prime \prime} \mathrm{N} ; 15^{\circ} 55^{\prime} 42^{\prime \prime} \mathrm{E}$. The sea depth was $1-3 \mathrm{~m}$ with the sea temperature at $15^{\circ} \mathrm{C}$. An air-tight plastic bag containing seawater and collected alga was transported to the laboratory immediately after the collection and was kept in the dark at $4{ }^{\circ} \mathrm{C}$ for not more than $48 \mathrm{~h}$ until further analysis. A part of the collected sample of $C$. adhaerens was placed in the dark at the room temperature for 14 days for air-drying. Both fresh and air-dried samples were cut in small pieces before further analysis.

A part of $C$. adhaerens was freeze-dried for the procedures in the Sections 3.5 and 3.6. Before the freeze-drying, the sample was washed in water (5 times) and in deionised water (2 times), then it was cut in $5-10 \mathrm{~mm}$ slices and frozen at $-60^{\circ} \mathrm{C}$ in an ultra-low freezer (CoolSafe PRO, Labogene, Lillerød, Denmark) for $24 \mathrm{~h}$. The freeze-drying was performed under a high vacuum $(0.13-0.55 \mathrm{hPa})$ at $-30^{\circ} \mathrm{C}$ and $20^{\circ} \mathrm{C}$ as the primary and secondary drying temperatures for $24 \mathrm{~h}$.

\subsection{Headspace Solid-Phase Microextraction (HS-SPME)}

A part of the fresh sample was placed between two filter paper layers for few minutes to remove a part of the excess seawater. HS-SPME was performed with PAL Auto Sampler System (PAL RSI 85, CTC Analytics AG, Schlieren, Switzerland) using two SPME fibres covered with DVB/CAR/PDMS (divinylbenzene/carboxen/polydimethylsiloxane) or PDMS/DVB (polydimethylsiloxane/divinylbenzene). Both fibres were purchased from Supelco Co. (Bellefonte, PA, USA) and were conditioned prior to the extraction. Prepared samples $(1 \mathrm{~g})$ were placed into $20 \mathrm{~mL}$ glass vials sealed with stainless steel cap with polytetrafluorethylene (PTFE)/silicon septa. The method was set to equilibrate the sample at $60^{\circ} \mathrm{C}$ for $15 \mathrm{~min}$ and then extract the sample for $45 \mathrm{~min}$. The injector temperature was set to $250^{\circ} \mathrm{C}$ and the thermal desorption directly to the GC column was carried out for 6 min. HS-SPME was performed in triplicate. 


\subsection{Hydrodistillation (HD)}

Hydrodistillation (HD) was performed in a modified Clevenger apparatus for $2 \mathrm{~h}$. Pentane (Fluka, Merck KGaA, Darmstadt, Germany) and diethyl ether (J.T. Baker Inc., Bridgewater, NJ, USA) were used as the solvent trap in $v / v$ ratio 1:2 $(1 \mathrm{~mL})$. The prepared samples of fresh and air-dried C. adhaerens were used separately for HD. The volatile oil dissolved in the solvent trap was removed with a pipette, passed through the layer of $\mathrm{MgSO}_{4}$ in a small glass funnel and slowly concentrated by the slow flow of nitrogen until the volume of $0.2 \mathrm{~mL}$. $2 \mu \mathrm{L}$ were used for GC-MS analyses.

\subsection{Gas Chromatography Mass Spectrometry Analysis of VOCs}

The GC-MS analyses of isolated VOCs were carried out with an Agilent Technologies (Palo Alto, Santa Clara, CA, USA) gas chromatograph model 8890 equipped with a mass spectrometer detector model 5977E MSD (Agilent Technologies). The VOCs separation was achieved on HP-5MS capillary column $(30 \mathrm{~m} \times 0.25 \mathrm{~mm}, 0.25 \mu \mathrm{m}$ film thickness, Agilent Technologies, Palo Alto, Santa Clara, CA, USA). The GC conditions and the detailed procedure are described in our previously published papers [11-13]: the injector and detector temperatures were $250{ }^{\circ} \mathrm{C}$ and $300^{\circ} \mathrm{C}$ and the oven temperature was set up isothermal at $70^{\circ} \mathrm{C}$ for $2 \mathrm{~min}$. Temperature gradient was achieved increasing the temperature from $70-200{ }^{\circ} \mathrm{C}$ at $3{ }^{\circ} \mathrm{C} / \mathrm{min}$ then was held isothermally at $200^{\circ} \mathrm{C}$ for $15 \mathrm{~min}$. Split ratio was 1:50; carrier gas was helium (He at flow rate $1.0 \mathrm{~mL} / \mathrm{min}$ ). The MSD (EI mode) was operated at $70 \mathrm{eV}$, and the mass range was set from 30 to $300 \mathrm{amu}$. The identification of the compounds was based on the comparison of their retention indices (RI), determined relative to the retention times of $n$-alkanes $\left(\mathrm{C}_{9}-\mathrm{C}_{25}\right)$, with those reported in the literature (National Institute of Standards and Technology) and their mass spectra with the spectra from Wiley 9 (Wiley, New York, NY, USA) and NIST 17 (D-Gaithersburg) mass spectral libraries. The percentage composition of the samples was calculated using the normalisation method (without correction factors). The average component percentages in Tables 1 and 2. were calculated from GC-MS analyses of three replicates.

\subsection{Gas Chromatography Flame-Ionisation Detection Analysis of Fatty Acids}

Total lipids were extracted from freeze dried C. adhaerens sample (FdCa) using Folch method [51] with chloroform/methanol (2:1 $v / v)$. The detailed methodology of lipid extraction and preparation of FAMEs was described in our previous paper [11]. The separation of prepared FAMEs was performed on a Shimadzu GC-2010 Plus gas chromatograph equipped with a flame ionisation detector (FID) and fitted with an SH-FAMEWAX ${ }^{\mathrm{TM}}$ capillary column $(30 \mathrm{~m}, 0.32 \mathrm{~mm}$ ID and $0.25 \mu \mathrm{m} \mathrm{df})$. The injector and detector temperatures were set at $240{ }^{\circ} \mathrm{C}$ and $250{ }^{\circ} \mathrm{C}$, respectively. The injection volume was $2 \mu \mathrm{L}$ with a split ratio of 1:100. The GC oven program was as follows: $120^{\circ} \mathrm{C}$ hold for $5 \mathrm{~min}$, to $220^{\circ} \mathrm{C}$ at $5{ }^{\circ} \mathrm{C} / \mathrm{min}$, hold for $20 \mathrm{~min}$. Nitrogen was used as carrier gas, flowing at the constant flow rate of $1.26 \mathrm{~mL} / \mathrm{min}$. The identification of separated FAMEs was achieved based on the comparison of retention times with the retention times of certified reference standard (Supelco F.A.M.E. Mix, C4-C24, St. Louis, MO, USA) analysed under the same conditions. The results were expressed as the percentage of identified fatty acid on total fatty acids (\%).

\subsection{Fractionation by Solid-Phase Extraction (SPE)}

The freeze-dried C. adhaerens ( $\mathrm{FdCa})$ was extracted $(10 \mathrm{~mL} / \mathrm{g}$ solvent:solid ratio) three times with sonication (ultrasound-bath Elma, Elmasonic P $70 \mathrm{H}$, Singen, Germany; $37 \mathrm{kHz} / 50 \mathrm{~W}$ ) for $5 \mathrm{~min}$ applying methanol:dichloromethane (MeOH/DCM, 1:1, v/v). The obtained extract was evaporated under nitrogen (5.0, Messer, Zapresic, Croatia), and was mixed with C18 powder (40-63 $\mu \mathrm{m}$, Macherey-Nagel Polygoprep 60-50 C18, Fisher Scientific, Hampton, NH, USA). The obtained dry extract was then placed on an SPE cartridge (C18, particle size $40 \mu \mathrm{m}$, bed weight 1g, column capacity $6 \mathrm{~mL}$, Agilent Bond Elut, Waldbronn, Germany), which was previously conditioned with $\mathrm{MeOH}$ and ultrapure water. Then the sample was eluted by applying the solvents of decreasing polarity to obtain 
the fractions $\mathrm{F} 1$ to $\mathrm{F} 4$ as was done in our previous paper [11]: $\mathrm{F} 1$ (with $\mathrm{H}_{2} \mathrm{O}$ ), $\mathrm{F} 2$ (with $\mathrm{H}_{2} \mathrm{O} / \mathrm{MeOH}(1: 1, v / v)$ ), $\mathrm{F} 3$ (with $\left.\mathrm{MeOH}\right)$, and F4 (with $\mathrm{MeOH} / \mathrm{DCM}(1: 1, v / v)$ ). Targeted less polar compounds were eluted in F3 and F4 and were dried by SpeedVac (SPD1030, Thermo Scientific, Waltham, MA, USA) and stored at $4{ }^{\circ} \mathrm{C}$ in dark.

\subsection{Ultra High-Performance Liquid Chromatography-High-Resolution Mass Spectrometry (UHPLC-ESI-HRMS) of F3 and F4}

The UPLC-HRMS analyses were performed using an ExionLC AD system (AB Sciex, Concord, Canada) equipped with the ExionLC solvent delivery system, ExionLC AD Pump, ExionLC AD Degasser, ExionLC AD Column oven, ExionLC AD Autosampler and ExionLC Controller combined with quadrupole-time-of-flight (Q-TOF) mass spectrometer TripleTOF 6600+ (AB Sciex, Concord, Canada) with Duospray ion source. The analytical column used for chromatographic separations was Acquity UPLC BEH Phenyl-Hexyl, $2.1 \mathrm{~mm} \times 100 \mathrm{~mm}$, particle size $1.7 \mu \mathrm{m}$ (Waters, Milford, MA, USA). The column oven temperature was set at $30^{\circ} \mathrm{C}$ and the flow rate was set at $0.4 \mathrm{~mL} / \mathrm{min}$. The mobile phases were water (A) and acetonitrile (B) both containing $0.1 \%$ formic acid. After 0.6 min of isocratic condition with $2 \%$ of $B$, the elution program was applied as follows: $0.6-18.5 \mathrm{~min}$ (B linear gradient to $100 \%), 18.5-25 \mathrm{~min}(100 \% \mathrm{~B})$. The injection volume was $4 \mu \mathrm{L}$.

Mass spectrometry detection was conducted in the positive electrospray ionisation $\left(\mathrm{ESI}^{+}\right.$). Tandem (MS /MS) mass spectra were recorded using collision-induced dissociation (CID) in information-dependent acquisition (IDA) mode for precursor ions with the signal intensities above $200 \mathrm{cps}$ threshold. The maximum number of precursor ions simultaneously subjected to CID was 15 . The ion source parameters were: nebulising gas (air, gas 1) pressure $40 \mathrm{psi}$, heater gas (air, gas 2) pressure $15 \mathrm{psi}$, curtain gas (nitrogen) pressure $30 \mathrm{psi}$, ESI capillary voltage $5.5 \mathrm{kV}$ and the source temperature $300{ }^{\circ} \mathrm{C}$. The recording mass spectra parameters were: declustering potential $80 \mathrm{~V}, \mathrm{~m} / \mathrm{z}$ range 100-1000 (MS) and 20-1000 (MS/MS), and accumulation time $100 \mathrm{~ms}$. The collision gas was nitrogen with the collision energy $40 \mathrm{eV}$ with a spread of $20 \mathrm{eV}$. The mass scale calibrations (in the MS and MS/MS modes) were done prior to each run in an automatic regime using a Tuning Solution (AB Sciex, Concord, Canada).

The data were processed using ACD/Spectrus Processor 2021.1.0. (ACD/Labs, Toronto, Canada). The elemental compositions of the compounds were determined based on the accurate masses of the corresponding protonated molecules, their isotopic distributions, and the product ions $m / z$ in MS/MS spectra. The tentative identification of detected components was carried out on the basis of their elemental compositions, tandem mass spectra and search in the ChemSpider database with a further selection of hits matching with MS/MS data.

\subsection{Antioxidant Activity of Tested Fractions by In Vitro Assays}

In vitro determination of antioxidant activity in this study employed four methods, including Folin-Ciocalteu method, ferric reducing antioxidant power (FRAP), reduction of the radical cation (ABTS) and 2,2-diphenyl-1-picryl-hydrazyl-hydrate (DPPH) assay. The measurements were carried out using a UV/Vis microplate reader (Infinite M200 PRO, TECAN, Männedorf, Switzerland) in multi-well plates (96-well) in triplicate. The results for all assays are expressed as mean \pm standard deviation $(n=3)$. All four mentioned methods were conducted in accordance with our previous research [11]. Briefly, obtained fractions were tested for their antioxidant activity by the reactions with appropriate reagents, incubated for a known period and change in colour with regards to control or blank sample was measured. Additionally, $\mathrm{IC}_{50}$ curve was obtained for both $\mathrm{F} 3$ and $\mathrm{F} 4$ by implementing the ABTS assay.

\subsection{Zebrafish Embryotoxicity Test (ZET)}

A breeding stock of healthy mature wild-type strain zebrafish Danio rerio (original supplier: European Zebrafish Resource Center of the Karlsruhe Institute of Technology 
(KIT), Karlsruhe, Germany) was used within this research. Zebrafish maintenance and embryo production was described in the paper of Babić et al. (2021) [52].

ZET was performed in accordance with OECD Test Guideline, in detail described in our previous study [11]. The fractions were tested in three concentrations (500, 250 and $125 \mu \mathrm{g} / \mathrm{mL}$ ) using 10 embryos in three replicates. Final solvent concentration (F3: MeOH; F4: DMSO) did not exceed $1 \%$. As a negative control artificial water was used, while $1 \%$ of $\mathrm{MeOH}$ and DMSO was tested as the solvent control. At $96 \mathrm{~h}$ of exposure, mortality and abnormality rates were recorded using an inverted microscope (Olympus CKX41) equipped with Leica EC3 digital camera and LAS EZ 3.2.0 digitising software.

\subsection{Antioxidant Effect of Tested Fractions Using Zebrafish Model}

The evaluation of the protective effect of $\mathrm{F} 3$ and $\mathrm{F} 4$ against $\mathrm{H}_{2} \mathrm{O}_{2}$-induced oxidative stress was conducted following the protocol described in our previous study [11]. Briefly, since no toxicity was recorded when performing the ZET test, zebrafish embryos were pre-treated with F3 and F4 in the concentration of 500, 250 and $125 \mu \mathrm{g} / \mathrm{mL}$ for $2 \mathrm{~h}$. After pre-treatment, oxidative stress was initiated with the addition of $5 \mathrm{mM} \mathrm{H}_{2} \mathrm{O}_{2}$. Upon $96 \mathrm{~h}$ of the exposure, the mortality rate was recorded, and survived specimens were stained with $10 \mu \mathrm{M}$ of fluorogenic dye dichloro-dihydro-fluorescein diacetate (DCF-DA) [11]. Intracellular ROS level in zebrafish larvae was visualised using a fluorescent microscope (Olympus ${ }^{\circledR}$ BX51 light binocular microscope; Microsoft ${ }^{\circledR}$ AnalySIS Soft Imaging System Software) with a green fluorescent filter. The fluorescence intensity of images was quantified using ImageJ software.

Statistical analysis and graphical representation were performed using GraphPad Prism version 8.0.1 for Windows, GraphPad Software, San Diego, CA, USA, www.graphpad. com accessed on 26 August 2021. One-way analysis of variance (ANOVA) and Tukey's post hoc test were performed to examine the significance between negative control and tested samples, as well as among treatments. When the assumption for normality was violated the Kruskal-Wallis one-way analysis of variance on ranks was performed. Student's paired $t$-test was chosen to analyse the data obtained with in vitro assays. The results were expressed as means $\pm \mathrm{SD}$, and $p \leq 0.05$ was used as a cut-off value of statistical significance throughout the paper.

\section{Conclusions}

C. adhaerens from the Adriatic Sea (Croatia) was comprehensively investigated regarding less polar compounds for the first time. Although there are several phytochemical studies of $C$. adhaerens from other regions, this is the first report on the volatile organic compounds (VOCs) from both fresh ( $\mathrm{FrCa}$ ) and air-dried ( $\mathrm{DrCa})$ samples. Great variability among HS-FrCa and HD-FrCa, as well as among HS-DrCa and HD-DrCa, was noted, as was expected according to the previous studies on different algae. This variability is the consequence of different applied methods isolating more and less volatile compounds as well as air-drying. However, the composition of HD and HS was partially similar to our previous study on C. bursa, indicating the same major VOCs (DMS and heptadecane) as chemical markers of Codium sp. DMS was the major compound in HS-FrCa. The oxidation of DMS to DMSO was noticed during air-drying. In HS-DrCa the majority of the identified compounds belong to alkanes with heptadecane as dominant. In HS-DrCa, three organoiodines were identified: iodomethane, diiodomethane and 1-iodopentane confirming the release of iodinated compounds under oxidative stress. The majority of VOCs in HD-FrCa and HD-DrCa belong to aliphatic compounds. The prevalent compound in HDFrCa was heptadecane, which decreased in HD-DrCa. Both chlorophyll derivatives and carotenoid degradation products (norisoprenoids) showed significant increase in HD-DrCa. The percentage of $(E)$-phytol and its oxidative product phytone increased during air-drying, indicating chlorophyll degradation. In $\mathrm{HD}-\mathrm{DrCa}$, two $\mathrm{C}_{10}$-norisoprenoids (safranal and $\beta$-cyclocitral) and one more $C_{13}$-norisoprenoid ( $\beta$-ionone) were identified, confirming oxidative cleavage of carotenoids and further cleavage of norisoprenoid compounds. 
Considering the $\omega 6$ FAs: $\omega 3$ FAs ratio, $C$. adhaerens can be considered a good source of dietary PUFAs. The $\omega 6$ fatty acids were present in higher content than $\omega 3$ fatty acids, as was found in previous studies, and the most abundant fatty acids were palmitic and arachidic acids.

The less polar fractions F3 and F4 were analysed by (UHPLC-ESI(+)-HRMS) for the first time. The major identified compounds were less polar compounds: chlorophyll derivatives (two sub-groups contacting 55 or 35 carbon atoms, mainly pheophytin $a$, pheophorbide $a$ and their derivatives), fatty acid glycerides, terpenes, steroids, and carotenoids.

The novelty of the present research is also related to in vitro and in vivo research on targeted antioxidant potential of less polar compounds from C. adhaerens. When compared, the results obtained by in vivo correlate well with in vitro methods and both fractions exerted similar antioxidative responses which is in agreement with the presence of a high abundance of biomolecules with known antioxidant properties (e.g., fucoxanthin, pheophytin $a$, and pheophorbide $a$ ). The results obtained using in vitro and in vivo approaches showed that the tested F3 and F4 fractions, in particular the chemical composition of $C$. adhaerens from the Adriatic Sea, exhibited high antioxidant activity and protective effects against $\mathrm{H}_{2} \mathrm{O}_{2}$-induced mortality of zebrafish embryos. These results suggest that $C$. adhaerens might be a potent source of natural antioxidants that could be used in research of oxidative stress-related diseases associated with the excess generation of reactive oxygen species such as cancer, cardiovascular and neurodegenerative diseases and therefore further research of this alga is encouraged.

Author Contributions: Conceptualisation, I.J., S.J. and S.B.; methodology, S.R., A.-M.C., I.F., S.B. and L.Č.; formal analysis, I.J., S.R., S.J., R.Č.-R. and D.Š.; investigation, R.Č-R., I.J. and S.J.; resources, S.R., I.J., S.B. and L.Č.; data curation, I.J., S.J., S.R., S.B., L.Č.; writing—original draft preparation, I.J., S.J., D.Š., R.Č.-R.; writing-review and editing, I.J., S.J. and R.Č.-R.; supervision, R.Č.-R. funding acquisition. All authors have read and agreed to the published version of the manuscript.

Funding: This research was funded by the Croatian Government and the European Union through the European Regional Development Fund-the Competitiveness and Cohesion Operational Programme (KK.01.1.1.01) through the project Bioprospecting of the Adriatic Sea (K.K.01.1.1.01.001) granted to The Scientific Centre of Excellence for Marine Bioprospecting-BioProCro.

Institutional Review Board Statement: Animal housing and spawning were performed in aquaria units approved by the Croatian Ministry of Agriculture and according to the Directive 2010/63/EU. All experiments in this study were conducted on the non-protected embryonic stages (up to $96 \mathrm{hpf}$ ), which do not require permission by animal welfare commissions (Directive 2010/63/EU).

Informed Consent Statement: Not applicable.

Data Availability Statement: Data is contained within the article.

Acknowledgments: We would like to thank the Croatian Government and the European Union (European Regional Development Fund - the Competitiveness and Cohesion Operational ProgrammeKK.01.1.1.01) granted to The Scientific Centre of Excellence for Marine Bioprospecting-BioProCro. We thank also Donat Petricioli for the sample collection and identification.

Conflicts of Interest: The authors declare no conflict of interest. The funders had no role in the design of the study; in the collection, analyses, or interpretation of data; in the writing of the manuscript, or in the decision to publish the results.

\section{References}

1. Ibñez, E.; Cifuentes, A. Benefits of using algae as natural sources of functional ingredients. J. Sci. Food Agric. 2013, 93, 703-709. [CrossRef] [PubMed]

2. Wang, L.; Wang, X.; Wu, H.; Liu, R. Overview on biological activities and molecular characteristics of sulfated polysaccharides from marine green algae in recent years. Mar. Drugs 2014, 12, 4984-5020. [CrossRef] [PubMed]

3. Rengasamy, K.R.R.; Mahomoodally, M.F.; Aumeeruddy, M.Z.; Zengin, G.; Xiao, J.; Kim, D.H. Bioactive compounds in seaweeds: An overview of their biological properties and safety. Food Chem. Toxicol. 2020, 135, 111013. [CrossRef]

4. Vinayak, R.C.; Sudha, S.A.; Chatterji, A. Bio-screening of a few green seaweeds from India for their cytotoxic and antioxidant potential. J. Sci. Food Agric. 2011, 91, 2471-2476. [CrossRef] 
5. Gutiérrez-Rodríguez, A.G.; Juárez-Portilla, C.; Olivares-Bañuelos, T.; Zepeda, R.C. Anticancer activity of seaweeds. Drug Discov. Today 2018, 23, 434-447.

6. Andrade, P.B.; Barbosa, M.; Matos, R.P.; Lopes, G.; Vinholes, J.; Mouga, T.; Valentão, P. Valuable compounds in macroalgae extracts. Food Chem. 2013, 138, 1819-1828. [CrossRef]

7. Yokohama, Y.; Kageyama, A.; Ikawa, T.; Shimura, S. A carotenoid characteristic of chlorophycean seaweeds living in deep coastal waters. Bot. Mar. 1977, 20, 433-436. [CrossRef]

8. Miyazawa, K.; Ito, K.; Matsumoto, F. Amino acids and peptide in seven species of marine green algae. J. Fac. Appl. Biol. Sci. Hiroshima Univ. 1976, 15, 161-169.

9. Lopes, G.; Sousa, C.; Bernardo, J.; Andrade, P.B.; Valentão, P. Sterol profiles in 18 macroalgae of the portuguese coast. J. Phycol. 2011, 47, 1210-1218. [CrossRef]

10. Lee, J.B.; Hayashi, K.; Maeda, M.; Hayashi, T. Antiherpetic activities of sulfated polysaccharides from green algae. Planta Med. 2004, 70, 813-817. [CrossRef] [PubMed]

11. Jerković, I.; Cikoš, A.-M.; Babić, S.; Čižmek, L.; Bojanić, K.; Aladić, K.; Ul’yanovskii, N.V.; Kosyakov, D.S.; Lebedev, A.T.; Čož-Rakovac, R.; et al. Bioprospecting of less-polar constituents from endemic brown macroalga Fucus virsoides J. Agardh from the Adriatic Sea and targeted antioxidant effects in vitro and in vivo (Zebrafish Model). Mar. Drugs 2021, 19, 235. [CrossRef] [PubMed]

12. Jerković, I.; Kranjac, M.; Marijanović, Z.; Šarkanj, B.; Cikoš, A.-M.; Aladić, K.; Pedisić, S.; Jokić, S. Chemical diversity of Codium bursa (Olivi) C. Agardh headspace compounds, volatiles, fatty acids and insight into its antifungal activity. Molecules 2019, $24,842$. [CrossRef] [PubMed]

13. Jerković, I.; Marijanović, Z.; Roje, M.; Kuś, P.M.; Jokić, S.; Čož-Rakovac, R. Phytochemical study of the headspace volatile organic compounds of fresh algae and seagrass from the Adriatic Sea (single point collection). PLoS ONE 2018, 13, e0196462.

14. Malin, G.; Steinke, M. Dimethyl sulfide production: What is the contribution of the coccolithophores? In Coccolithophores; Thierstein, H.R., Young, J.R., Eds.; Springer: Berlin/Heidelberg, Germany, 2004; pp. 127-164.

15. Alcolombri, U.; Ben-Dor, S.; Feldmesser, E.; Levin, Y.; Tawfik, D.S.; Vardi, A. Identification of the algal dimethyl sulfide-releasing enzyme: A missing link in the marine sulfur cycle. Science 2015, 348, 1466-1469. [CrossRef]

16. Estevez, J.M.; Fernández, P.V.; Kasulin, L.; Dupree, P.; Ciancia, M. Chemical and in situ characterization of macromolecular components of the cell walls from the green seaweed Codium fragile. Glycobiology 2009, 19, 212-228. [CrossRef]

17. Domozych, D.; Ciancia, M.; Fangel, J.U.; Mikkelsen, M.D.; Ulvskov, P.; Willats, W.G. The cell walls of green algae: A journey through evolution and diversity. Front. Plant Sci. 2012, 3, 8. [CrossRef]

18. Rezanka, T.; Zahradník, J.; Podojil, M. Hydrocarbons in green and blue-green algae. Folia Microbiol. 1982, 27, 450-454. [CrossRef]

19. Mironov, O.G.; Shchekaturina, T.L.; Tsimbal, I.M. Saturated hydrocarbons in marine organisms. Mar. Ecol. Prog. Ser. 1981, 5, 303-309. [CrossRef]

20. Han, J.; Chan, H.W.-S.; Calvin, M. Biosynthesis of alkanes in Nostoc muscorum. J. Am. Chem. Soc. 1969, 91, 5156-5159. [CrossRef]

21. Moore, R.E. Volatile compounds from marine algae. Acc. Chem. Res. 1977, 10, 40-47. [CrossRef]

22. Palmer, C.J.; Anders, T.L.; Carpenter, L.J.; Küpper, F.C.; McFiggans, G.B. Iodine and halocarbon response of Laminaria digitata to oxidative stress and links to atmospheric new particle production. Environ. Chem. 2006, 2, 282-290. [CrossRef]

23. Bravo-Linares, C.M.; Mudge, S.M.; Loyola-Sepulveda, R.H. Production of volatile organic compounds (VOCs) by temperate macroalgae: The use of Solid Phase Microextraction (SPME) coupled to GC-MS as method of analysis. J. Chilean Chem. Soc. 2010, 55, 227-232. [CrossRef]

24. Franssen, M.C.R. Halogenation and oxidation reactions with haloperoxidases. Biocatalysis 1994, 10, 87-111. [CrossRef]

25. Nozaki, H. Flagellated green algae. In Freshwater Algae of North America: Ecology and Classification, 1st ed.; Wehr, J.D., Sheath, R.G., Eds.; Academic Press: London, UK, 2003; pp. 225-252.

26. Gutbrod, P.; Yang, W.; Grujicic, G.V.; Peisker, H.; Gutbrod, K.; Du, L.F.; Dörmann, P. Phytol derived from chlorophyll hydrolysis in plants is metabolized via phytenal. J. Biol. Chem. 2021, 296, 100530. [CrossRef]

27. Rodríguez-Bustamante, E.; Sánchez, S. Microbial production of $\mathrm{C}_{13}$-norisoprenoids and other aroma compounds via carotenoid cleavage. Crit. Rev. Microbiol. 2007, 33, 211-230. [CrossRef]

28. Pereira, H.; Barreira, L.; Figueiredo, F.; Custódio, L.; Vizetto-Duarte, C.; Polo, C.; Rešek, E.; Engelen, A.; Varela, J. Polyunsaturated fatty acids of marine macroalgae: Potential for nutritional and pharmaceutical applications. Mar. Drugs 2012, 10, 1920-1935. [CrossRef]

29. Goecke, F.; Hernández, V.; Bittner, M.; González, M.; Becerra, J.; Silva, M. Fatty acid composition of three species of Codium (Bryopsidales, Chlorophyta) in Chile. Rev. Biol. Mar. Oceanogr. 2010, 45, 325-330. [CrossRef]

30. van Ginneken, V.J.T.; Helsper, J.P.F.G.; de Visser, W.; van Keulen, H.; Brandenburg, W.A. Polyunsaturated fatty acids in various macroalgal species from north Atlantic and tropical seas. Lipids Health Dis. 2011, 10, 104. [CrossRef]

31. Kuczynska, P.; Jemiola-Rzeminska, M.; Strzalka, K. Photosynthetic pigments in diatoms. Mar. Drugs 2015, 13, 5847-5881. [CrossRef] [PubMed]

32. Okai, Y.; Higashi-Okai, K. Potent anti-inflammatory activity of pheophytin a derived from edible green alga, enteromorpha prolifera (sujiao-nori). Int. J. Immunopharmacol. 1997, 19, 355-358. [CrossRef]

33. Okai, Y.; Ishizaka, S.; Higashi-Okai, K.; Yamashita, U. Detection of immunomodulating activities in an extract of Japanese edible seaweed Laminaria japonica (Makonbu). J. Sci. Food Agric. 1996, 72, 455-460. [CrossRef] 
34. Cahyana, A.H.; Shuto, Y.; Kinoshita, Y. Pyropheophytin $a$ as an antioxidative substance from the marine alga Arame (Eisenis bicyclis). Biosci. Biotech. Biochem. 1992, 56, 1533-1535. [CrossRef]

35. Nishibori, S.; Namiki, K. Antioxidative substances in the green fractions of the lipid of Aonori (Enteromorpha sp.). J. Home Econ. 1998, 39, 1173-1178.

36. Saide, A.; Lauritano, C.; Ianora, A. Pheophorbide $a$ : State of the Art. Mar. Drugs 2020, 18, 257. [CrossRef] [PubMed]

37. Ochi, M.; Watanabe, M.; Miura, I.; Taniguchi, M.; Tokoroyama, T. Amijiol, isoamijiol, and 14-deoxyamijiol, three new diterpenoids from the brown seaweed Dictyota linearis. Chem. Lett. 1980, 9, 1229-1232. [CrossRef]

38. Matsuno, T. Aquatic animal carotenoids. Fish. Sci. 2001, 67, 771-783. [CrossRef]

39. Maeda, H.; Fukuda, S.; Izumi, H.; Saga, N. Anti-oxidant and fucoxanthin contents of brown alga ishimozuku (Sphaerotrichia divaricata) from the west coast of Aomori, Japan. Mar. Drugs 2018, 16, 255. [CrossRef]

40. Méresse, S.; Fodil, M.; Fleury, F.; Chénais, B. Fucoxanthin, a marine-derived carotenoid from brown seaweeds and microalgae: A promising bioactive compound for cancer therapy. Int. J. Mol. Sci. 2020, 21, 9273. [CrossRef]

41. Cikoš, A.-M.; Flanjak, I.; Bojanić, K.; Babić, S.; Čižmek, L.; Čož-Rakovac, R.; Jokić, S.; Jerković, I. Bioprospecting of coralline red alga Amphiroa rigida J.V. Lamouroux: Volatiles, fatty acids and pigments. Molecules 2021, 26, 520. [CrossRef]

42. Pangestuti, R.; Kim, S.-K. Biological activities and health benefit effects of natural pigments derived from marine algae. J. Funct. Foods 2011, 3, 255-266. [CrossRef]

43. Sudha, K.; Mohana Priya, K.; Nancy Veena, K.; Palanichamy, V. Screening of antioxidant potential of green alga Codium adhaerens. Int. J. Drug Dev. Res. 2014, 6, 103-111.

44. Foo, S.C.; Yusoff, F.M.; Ismail, M.; Basri, M.; Yau, S.K.; Khong, N.M.H.; Chan, K.W.; Ebrahimi, M. Antioxidant capacities of fucoxanthin-producing algae as influenced by their carotenoid and phenolic contents. J. Biotechnol. 2017, 241, 175-183. [CrossRef] [PubMed]

45. Pinteus, S.; Silva, J.; Alves, C.; Horta, A.; Fino, N.; Rodrigues, A.I.; Mendes, S.; Pedrosa, R. Cytoprotective effect of seaweeds with high antioxidant activity from the Peniche coast (Portugal). Food Chem. 2017, 1, 591-599. [CrossRef]

46. Horzmann, K.A.; Freeman, J.L. Making waves: New developments in toxicology with the zebrafish. Toxicol. Sci. 2018, 163, 5-12. [CrossRef]

47. Kang, M.C.; Lee, S.H.; Lee, W.W.; Kang, N.; Kim, E.A.; Kim, S.Y.; Lee, D.H.; Kim, D.; Jeon, Y.-J. Protective effect of fucoxanthin isolated from Ishige okamurae against high-glucose inducedoxidative stress in human umbilical veinendothelial cells and zebrafish model. J. Funct. Foods 2014, 11, 304-312. [CrossRef]

48. Hsu, C.Y.; Chao, P.Y.; Hu, S.P.; Yang, C.M. The antioxidant and free radical scavenging activities of chlorophylls and pheophytins. Food Sci. Nutr. 2013, 4, 1-8. [CrossRef]

49. Lee, H.; Park, H.Y.; Jeong, T.S. Pheophorbide $a$ derivatives exert antiwrinkle effects on UVB-induced skin aging in human fibroblasts. Life 2021, 11, 147. [CrossRef] [PubMed]

50. Hsu, C.Y.; Yang, C.M.; Chen, C.M.; Chao, P.Y.; Hu, S.P. Effects of chlorophyll-related compounds on hydrogen peroxide induced DNA damage within human lymphocytes. J. Agric. Food Chem. 2005, 53, 2746-2750. [CrossRef]

51. Folch, J.; Lees, M.; Stanley, G.H.S. A simple method for the isolation and purification of total lipides from animal tissues. J. Biol. Chem. 1957, 226, 497-509. [CrossRef]

52. Babić, S.; Čižmek, L.; Maršavelski, A.; Malev, O.; Pflieger, M.; Strunjak-Perović, I.; Topić Popović, N.; Čož-Rakovac, R.; Trebše, P. Utilization of the zebrafish model to unravel the harmful effects of biomass burning during Amazonian wildfires. Sci. Rep. 2021, 11, 1-12. [CrossRef] 